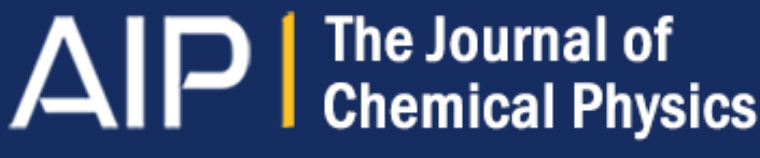

Electron collisions with phenol: Total, integral, differential, and momentum transfer cross sections and the role of multichannel coupling effects on the elastic channel

Romarly F. da Costa, Eliane M. de Oliveira, Márcio H. F. Bettega, Márcio T. do N. Varella, Darryl B. Jones, Michael J. Brunger, Francisco Blanco, Rafael Colmenares, Paulo Limão-Vieira, Gustavo García, and Marco A. P. Lima

Citation: The Journal of Chemical Physics 142, 104304 (2015); doi: 10.1063/1.4913824

View online: http://dx.doi.org/10.1063/1.4913824

View Table of Contents: http://scitation.aip.org/content/aip/journal/jcp/142/10?ver=pdfcov

Published by the AIP Publishing

\section{Articles you may be interested in}

Cross Sections for Electron Collisions with Methane

J. Phys. Chem. Ref. Data 44, 023101 (2015); 10.1063/1.4918630

Integral cross sections for electron impact excitation of vibrational and electronic states in phenol

J. Chem. Phys. 142, 194305 (2015); 10.1063/1.4921313

Cross Sections for Electron Collisions with Carbon Monoxide

J. Phys. Chem. Ref. Data 44, 013105 (2015); 10.1063/1.4913926

Total, elastic, and inelastic cross sections for positron and electron collisions with tetrahydrofuran

J. Chem. Phys. 138, 074301 (2013); 10.1063/1.4789584

Elastic and inelastic cross sections for low-energy electron collisions with pyrimidine

J. Chem. Phys. 136, 144310 (2012); 10.1063/1.3702629

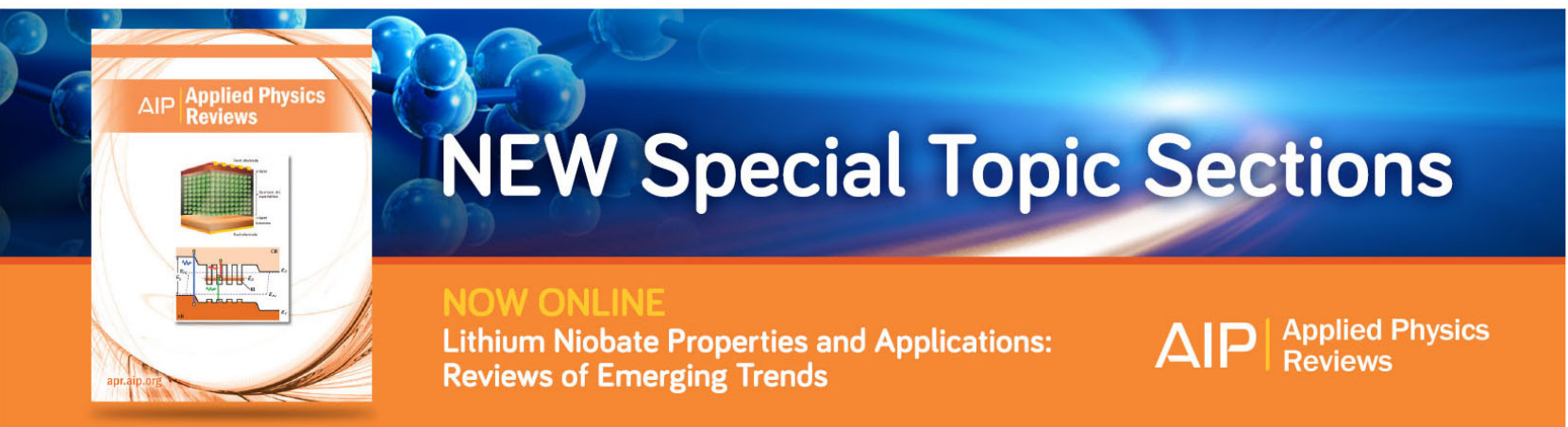




\title{
Electron collisions with phenol: Total, integral, differential, and momentum transfer cross sections and the role of multichannel coupling effects on the elastic channel
}

\author{
Romarly F. da Costa, ${ }^{1,2}$ Eliane M. de Oliveira, ${ }^{1}$ Márcio H. F. Bettega, ${ }^{3}$ \\ Márcio T. do N. Varella, ${ }^{4}$ Darryl B. Jones, ${ }^{5}$ Michael J. Brunger, ${ }^{5,6}$ Francisco Blanco, ${ }^{7}$ \\ Rafael Colmenares, ${ }^{8}$ Paulo Limão-Vieira, ${ }^{9}$ Gustavo García, ${ }^{10}$ and Marco A. P. Lima ${ }^{1}$ \\ ${ }_{1}^{1}$ Instituto de Física "Gleb Wataghin," Universidade Estadual de Campinas, \\ 13083-859 Campinas, São Paulo, Brazil \\ ${ }^{2}$ Centro de Ciências Naturais e Humanas, Universidade Federal do ABC, \\ 09210-580 Santo André, São Paulo, Brazil \\ ${ }^{3}$ Departamento de Física, Universidade Federal do Paraná, CP 19044, 81531-990 Curitiba, Paraná, Brazil \\ ${ }^{4}$ Instituto de Física, Universidade de São Paulo, CP 66318, 05315-970 São Paulo, São Paulo, Brazil \\ ${ }^{5}$ School of Chemical and Physical Sciences, Flinders University, GPO Box 2100, Adelaide SA 5001, Australia \\ ${ }^{6}$ Institute of Mathematical Sciences, University of Malaya, 50603 Kuala Lumpur, Malaysia \\ ${ }^{7}$ Departamento de Fisica Atomica, Molecular y Nuclear, Universidad Complutense de Madrid, \\ Ciudad Universitaria, 2840 Madrid, Spain \\ ${ }^{8}$ Hospital Ramón y Cajal, 28034 Madrid, Spain \\ ${ }^{9}$ Laboratório de Colisões Atómicas e Moleculares, CEFITEC, Departamento de Física, Faculdade de Ciências \\ e Tecnologia, Universidade Nova de Lisboa, 2829-516 Caparica, Portugal \\ ${ }^{10}$ Instituto de Fisica Fundamental, CSIC, Serrano 113-bis, 28006 Madrid, Spain
}

(Received 23 December 2014; accepted 18 February 2015; published online 10 March 2015)

\begin{abstract}
We report theoretical and experimental total cross sections for electron scattering by phenol $\left(\mathrm{C}_{6} \mathrm{H}_{5} \mathrm{OH}\right)$. The experimental data were obtained with an apparatus based in Madrid and the calculated cross sections with two different methodologies, the independent atom method with screening corrected additivity rule (IAM-SCAR), and the Schwinger multichannel method with pseudopotentials (SMCPP). The SMCPP method in the $N_{\text {open }}$-channel coupling scheme, at the static-exchange-plus-polarization approximation, is employed to calculate the scattering amplitudes at impact energies ranging from $5.0 \mathrm{eV}$ to $50 \mathrm{eV}$. We discuss the multichannel coupling effects in the calculated cross sections, in particular how the number of excited states included in the open-channel space impacts upon the convergence of the elastic cross sections at higher collision energies. The IAM-SCAR approach was also used to obtain the elastic differential cross sections (DCSs) and for correcting the experimental total cross sections for the so-called forward angle scattering effect. We found a very good agreement between our SMCPP theoretical differential, integral, and momentum transfer cross sections and experimental data for benzene (a molecule differing from phenol by replacing a hydrogen atom in benzene with a hydroxyl group). Although some discrepancies were found for lower energies, the agreement between the SMCPP data and the DCSs obtained with the IAM-SCAR method improves, as expected, as the impact energy increases. We also have a good agreement among the present SMCPP calculated total cross section (which includes elastic, 32 inelastic electronic excitation processes and ionization contributions, the latter estimated with the binary-encounter-Bethe model), the IAM-SCAR total cross section, and the experimental data when the latter is corrected for the forward angle scattering effect [Fuss et al., Phys. Rev. A 88, 042702 (2013)]. (C) 2015 AIP Publishing LLC. [http://dx.doi.org/10.1063/1.4913824]
\end{abstract}

\section{INTRODUCTION}

One of the relevant challenges for a theoretician working on electron-molecule scattering calculations consists of defining a proper balance between the open- and closed-channel electronic space, for description of the $(N+1)$-electron problem to be solved. Separation of the nuclear motion from the scattering dynamics caused by the electronic cloud in a fixednuclei approximation is the usual strategy to simplify the problem. Even at this level of approximation, in molecular targets, the density of electronically excited states is usually so large that the convergence of multichannel scattering calculations could be seriously hindered in many cases of practical interest. This is particularly true for molecules having its first thresholds opening up at 3-4 eV (as is the case of the DNA bases) and becomes even more critical as the energy of the incident electron increases. In such cases it is therefore necessary to judiciously truncate the electronic configuration space. At sufficiently low energies, only the elastic channel is open and the distortion of the electronic cloud is taken into account by allowing virtual excitations (closed channel space) from the ground state. This effect combined with the proper solution of the scattering problem, including the exchange interaction, is known as static-exchange-plus-polarization (SEP) 
approximation. As we increase the impact energy, several discrete and continuum states (above the ionization threshold) can be excited giving rise to the important questions: (1) how many of these states must be included in a calculation in order to provide a reasonable approximation of the experimental total cross section (TCS)? (2) how do these multichannel effects affect the elastic differential cross sections (DCSs) and their related integral and momentum transfer cross sections (MTCS)? (3) how do they affect the other electronic excitation cross sections? and (4) how sophisticated must the electronic states description be in order to accurately calculate the cross sections? Recent applications ${ }^{1-4}$ performed by theoretical groups using different methods for describing the electronic excitation of molecules by electron impact, at specific levels of multichannel coupling, have addressed one or more of these issues. They clearly indicate that there are many challenges that must be overcome to obtain reliable cross sections. On the experimental side, the energy separation of the electronic states is the main challenge. It is thus very common to work with unresolved states, particularly in polyatomic species, and pursue measurements for bands containing a few or more states related to a particular energy loss range.

In this paper, we present a theoretical and experimental effort to obtain cross sections for scattering of low and intermediate energy electrons by the phenol molecule. Phenol is an interesting molecule for technological applications ${ }^{5}$ and in addition it contains several characteristics that make this joint effort relevant: (1) it is a small complex organic molecule, (2) it has a high density of electronic states at relatively small impact energies, and (3) it has a permanent dipole moment, which is always a challenge for both total cross section measurements and the calculations. The present paper is outlined as follows. In Secs. II-V, different aspects of our theoretical models and the corresponding computational details are briefly described. In Sec. VI, experimental details of the apparatus in Madrid, used to obtain the present total cross sections, are described. Results and a discussion of these results are subsequently presented in Secs. VII and VIII, with a small comment on the choice of $\ell_{\max }$ in the calculations being given in Sec. IX. Finally, in Sec. X, some concluding remarks from this investigation are summarized.

\section{THE SCHWINGER MULTICHANNEL METHOD WITH PSEUDOPOTENTIALS (SMCPP)}

The Schwinger multichannel method (SMC) ${ }^{6}$ is a variational approach that uses square integrable basis functions to obtain the scattering amplitude for electron-molecule collisions, including important effects such as exchange, polarization, and electronic multichannel coupling. In the present calculations, we use the parallel version ${ }^{7}$ of the SMC implementation that employs norm-conserving pseudopotentials ${ }^{8}$ (SMCPP) and single-excitation configuration interaction techniques for the target description. ${ }^{9}$ Since the method and its computational implementation were described in detail elsewhere, ${ }^{9}$ here we only give the working expression for the scattering amplitude

$$
f\left(\mathbf{k}_{f}, \mathbf{k}_{i}\right)=-\frac{1}{2 \pi} \sum_{m, n}\left\langle S_{\mathbf{k}_{f}}|V| \chi_{m}\right\rangle\left(d^{-1}\right)_{m n}\left\langle\chi_{n}|V| S_{\mathbf{k}_{i}}\right\rangle,
$$

where

$$
\begin{aligned}
d_{m n}= & \left\langle\chi_{m}\right|\left[\frac{\hat{H}}{N+1}-\frac{\hat{H} P+P \hat{H}}{2}\right. \\
& \left.+\frac{P V+V P}{2}-V G_{P}^{(+)} V\right]\left|\chi_{n}\right\rangle .
\end{aligned}
$$

In the expressions above, $P$ is a projector onto $N_{\text {open }}$ energyallowed target electronic channels, i.e.,

$$
P=\sum_{\ell=1}^{N_{\text {open }}}\left|\Phi_{\ell}\right\rangle\left\langle\Phi_{\ell}\right|
$$

$G_{P}^{(+)}$is the free-particle Green's function projected onto the $P$ space, $V$ is the projectile-target interaction potential, $\mathbf{k}_{i}$ $\left(\mathbf{k}_{f}\right)$ is the incoming (outgoing) projectile wave vector, and $\hat{H}=E-H$ is the total energy (ground state energy plus kinetic energy of the incoming electron) minus the Hamiltonian of the $(N+1)$ electrons under the field of the fixed nuclei. The latter is given by $H=H_{0}+V$, where $H_{0}$ describes the non-interacting electron-molecule system and $S_{\mathbf{k}}$ is a solution of $H_{0}$, namely, the product of a plane wave (projectile) and a target state $\Phi_{\ell}$ (obtained within the scope of the single excitation configuration-interaction description). For the expansion of the variational scattering wave function, the method employs trial basis sets comprising $(N+1)$-particle configuration state functions (CSFs), denoted by $\chi_{m}$, that are built from spin-adapted, anti-symmetrized products of target electronic states and projectile scattering orbitals. The open electronic collision channels are included in the $P$ space, and the dynamical response of the target electrons to the projectile field (correlation-polarization effects) is accounted for through virtual excitations of the target. In this case, the CSFs are given by

$$
\left|\chi_{m}\right\rangle=\mathcal{A}_{N+1}\left|\Phi_{i}(1, \ldots, N)\right\rangle \otimes\left|\varphi_{j}(N+1)\right\rangle,
$$

where for $i>0,\left|\Phi_{i}\right\rangle \equiv{ }^{(2 S+1)}\left(h_{i} \rightarrow p_{i}\right)$ is a singly excited state obtained by promoting one electron from a hole orbital $\left(h_{i}\right)$ of the ground state $\Phi_{0}(1, \ldots, N)$ to a particle orbital $\left(p_{i}\right)$, with either singlet $(S=0)$ or triplet $(S=1)$ spin coupling, though only $(N+1)$-electron configurations with total spin $S=1 / 2$ (doublets) are actually taken into account. If we have $N_{\text {open }}$ states in Eq. (3), this level of calculation is called an $N_{\text {open }}$-channel coupling scheme at the static-exchangeplus-polarization (acronym is $N_{\text {open }}$ ch-sep) approximation. In order to transform the scattering amplitude from the bodyfixed frame (the reference frame best suited for carrying out the calculations) to the laboratory-fixed frame (the reference frame where the $z$-axis is aligned with the direction of the incident wave vector, i.e., $\mathbf{k}_{i}=k_{i} \hat{\mathbf{z}}$ ), we expand $\mathbf{k}_{f}$ in terms of partial waves ${ }^{10}$

$$
f\left(\mathbf{k}_{f}, \mathbf{k}_{i}\right) \equiv\left\langle\mathbf{k}_{f}|f| \mathbf{k}_{i}\right\rangle=\sum_{\ell=0}^{\ell_{\max }} \sum_{m=-\ell}^{\ell}\left\langle\mathbf{k}_{f} \mid \ell m\right\rangle f\left(\ell m, \mathbf{k}_{i}\right),
$$

where $\left\langle\mathbf{k}_{f} \mid \ell m\right\rangle$ is a spherical harmonic that can be easily converted from the body- to the laboratory-frame and $f\left(\ell m, \mathbf{k}_{i}\right)$ $=\left\langle\ell m|f| \mathbf{k}_{i}\right\rangle$ can be understood as the scattering amplitude of an electron entering the interaction region in a plane-wave $\left|\mathbf{k}_{i}\right\rangle$ and leaving it in a partial wave $|\ell m\rangle$. Although not shown 
here, all SMCPP differential cross sections in this paper, over the entire energy range $(5-50 \mathrm{eV})$, are numerically converged with $\ell_{\max }=10$ (except for $50 \mathrm{eV}$ that demands $\ell_{\max }=13$ ) if combined with a quadrature point distribution, using a 26 Gauss-Legendre scheme for $0 \leq \theta_{i} \leq \pi$ and 52 points for $0 \leq \phi_{i} \leq 2 \pi$, to describe $\mathbf{k}_{i}\left(\theta_{i}, \phi_{i}\right)$ in spherical coordinates. Although we have contributions from high partial waves in the scattering orbitals due to the multi-center expansion, we only employ Cartesian Gaussians (CG) of $s, p$, and $d$ types on the oxygen atom and on each center for the carbon atoms. On the hydrogen atoms we have only CG functions of $s$ and $p$ types. As discussed in our previous applications, ${ }^{4}$ this makes the description of the high partial waves more difficult but sufficient to obtain good convergence in the elastic differential cross sections. We have also used pseudopotentials for the carbon and oxygen atoms. This strategy allows a reduction in the number of Cartesian Gaussian functions, since it is not necessary to consider those involved in the description of all $1 s$ orbitals of these atoms.

Another way of calculating the scattering amplitude is by also expanding $\mathbf{k}_{i}$ in partial waves as follows:

$$
\left\langle\mathbf{k}_{f}|f| \mathbf{k}_{i}\right\rangle=\sum_{l=0}^{\ell_{\max }} \sum_{m=-\ell}^{\ell} \sum_{l^{\prime}=0}^{\ell_{\max }} \sum_{m^{\prime}=-\ell^{\prime}}^{\ell^{\prime}}\left\langle\mathbf{k}_{f} \mid \ell m\right\rangle f\left(\ell m, \ell^{\prime} m^{\prime}\right)\left\langle\ell^{\prime} m^{\prime} \mid \mathbf{k}_{i}\right\rangle .
$$

The DCSs are obtained from $f\left(\ell m, \ell^{\prime} m^{\prime}\right)=\left\langle\ell, m|f| \ell^{\prime}, m^{\prime}\right\rangle$ which represents a scattering amplitude of an electron entering the interaction region in a partial wave $\left|\ell^{\prime}, m^{\prime}\right\rangle$ and leaving it in a partial wave $|\ell, m\rangle$. The expansion up to $\ell_{\max }=4$ is a standard procedure used in other scattering computer codes and, in order to allow future comparisons, we have included results calculated from Eq. (6) with several values of $\ell_{\text {max }}$. For some cases (elastic and dipole-allowed singlet transitions), a Born-closure scheme was used following the same strategy as described in Ref. 11. This closure is obtained from the expression

$$
\begin{aligned}
f_{\mathrm{LAB}}^{\text {closure }}\left(\mathbf{k}_{f}, \mathbf{k}_{i}\right)= & f_{\mathrm{LAB}}^{\mathrm{FBA}}\left(\mathbf{k}_{f}, \mathbf{k}_{i}\right)+\sum_{\ell=0}^{\ell_{\max }} \sum_{m=-\ell}^{\ell}\left(f_{\mathrm{LAB}}\left(\ell m, \mathbf{k}_{i}\right)\right. \\
& \left.-f_{\mathrm{LAB}}^{\mathrm{FBA}}\left(\ell m, \mathbf{k}_{i}\right)\right) Y_{\ell m}^{*}\left(\mathbf{k}_{f}\right),
\end{aligned}
$$

where $f_{\mathrm{LAB}}^{\mathrm{FBA}}$ is the scattering amplitude for the permanent dipole moment potential for the elastic process or for the dipole transition potential for inelastic dipole-allowed processes. Both are obtained in the first Born approximation, in a closed form in the laboratory-frame. The amplitude $f_{\mathrm{LAB}}\left(\ell m, \mathbf{k}_{i}\right)$ is just the $f\left(\ell m, \mathbf{k}_{i}\right)$ of Eqs. (5) or (6) transformed to this frame.

For the total cross section, we have used the optical theorem directly on the linear representation of the scattering amplitude, given by Eq. (1), i.e.,

$$
\sigma_{\text {tot }}=\frac{1}{4 \pi} \int d \Omega_{\mathbf{k}_{i}} \frac{4 \pi}{k} \operatorname{Im} f\left(\mathbf{k}_{i}, \mathbf{k}_{i}\right)=\sum_{n=1}^{N_{\text {open }}} \sigma(1 \rightarrow n),
$$

where $\sigma(1 \rightarrow n)$ is the integral cross section (ICS) for the electronic transition $1 \rightarrow n$. In order to correct the contribution from the low-angular region (important for the elastic transition since phenol has a non-negligible permanent dipole moment, and for singlet excitations to dipole-allowed states), we have also calculated the TCS, using the amplitudes obtained from Eq. (7), and the expression

$$
\sigma_{\text {tot }}^{\text {closure }}=\sum_{n=1}^{N_{\text {open }}} \sigma^{\text {closure }}(1 \rightarrow n),
$$

where $\sigma^{\text {closure }}(1 \rightarrow n) \neq \sigma(1 \rightarrow n)$ only for the above mentioned transitions.

We have also employed the binary-encounter-Bethe (BEB) model $^{12}$ to estimate the total ionization cross section by electron impact. This cross section, added to our computed elastic+inelastic cross section, will be compared to the total cross section data. This model provides the following expression for the ionization cross section per molecular orbital:

$$
\sigma_{B E B}(T)=\frac{S}{t+u+1}\left[\frac{\ln t}{2}\left(1-\frac{1}{t^{2}}\right)+1-\frac{1}{t}-\frac{\ln t}{t+1}\right] .
$$

In Eq. (10), $T$ is the incident electron energy, $t=T / B$ and $u$ $=U / B$ are normalized energies, where $B$ and $U$ are the orbital binding and electron kinetic energy, respectively, and $S$ $=4 \pi a_{0}^{2} N_{\text {occ }} R^{2} / B^{2}$, where $N_{\text {occ }}$ is the orbital occupation number, $a_{0}=0.5292 \AA$, and $R=13.61 \mathrm{eV}$. The total ionization cross section is obtained by the summation of $\sigma_{B E B}(T)$ over the molecular orbitals satisfying $T>B$. This model provides ionization cross sections which agree with experiment to within 5\%-15\% (considering different molecules) for incident energies ranging from the first ionization threshold to several $\mathrm{keV}^{13}$

\section{COMPUTATIONAL PROCEDURES OF THE PRESENT APPLICATION OF THE SMCPP METHOD}

In the present application of the SMCPP method, we have used the same Cartesian Gaussian basis set of Ref. 14 and the description of the target ground and excited states as in Ref. 15. This description can be summarized as (1) we have used 247 uncontracted Cartesian Gaussian functions for the description of target bound states and for the scattering calculations (CSFs basis of Eq. (4)), with $s, p$, and $d$ types on the $\mathrm{C}$ and $\mathrm{O}$ atoms, and $s$ and $p$ types on the $\mathrm{H}$ atoms; (2) as usual, ${ }^{14}$ a Hartree-Fock calculation was carried out for the ground state, giving a permanent dipole moment of $1.423 \mathrm{D}$ against the experimental value of $1.224 D$ of Ref. 16; (3) following the same strategy as used in our recent electronethylene multichannel study ${ }^{4}$ and as described in Ref. 15, a full single excitation configuration-interaction (FSCI) was obtained, ${ }^{17}$ so as to give excitation energies in reasonable agreement with the experimental spectra and time-dependent density functional theory calculations, ${ }^{15}$ particularly so for states lying below an arbitrary chosen energy of $7 \mathrm{eV}$; (4) a small set of singlet and triplet improved virtual orbitals were obtained and used as a minimum orbital basis for a single configuration interaction $(\mathrm{MOBSCI})^{9}$ calculation, that was capable of describing all states below $7 \mathrm{eV}$ in good agreement with the FSCI calculation. This was possible with 16 holeparticle pairs of single excitations, giving rise to 16 triplet 
and 16 singlet states with 7 triplets and 5 singlets below our arbitrary chosen $7 \mathrm{eV}$ energy.

The need for choosing a small value of energy $(7 \mathrm{eV})$ is due to the high cost of the scattering calculation, when considering too big a value of $N_{\text {open }}$ in Eq. (3) (for every electronic channel, a large numerical quadrature must be carried out in order to evaluate the projected Green's function ${ }^{18}$ of Eq. (2)). Our present CSF space for the scattering calculations has a smaller size than that used in our previous investigation ${ }^{14}$ and it was also constructed with modified virtual orbitals (in addition to the active particle orbitals of the MOBSCI) in order to better account for polarization effects. It is worthwhile noting that the configuration space is the same for all the present $N_{\text {open }}$ ch-sep approximations (that is, for calculations with $N_{\text {open }}=1,2 \ldots$ or 33 , where more collision channels are opened as the electron impact energy increases). It is also important to mention that the present 1ch-sep cross sections obtained with this procedure are in close agreement with those previously reported, ${ }^{14}$ highlighting that the effect which will be discussed arises mainly from the different channel coupling schemes used in the present study. We have used the acronym 1ch-sep for the elastic process only, 3ch-sep for the ground plus first two triplet states (all states assigned to the elastic channel plus Band 1 of Ref. 15), the 8ch-sep for the ground state plus 5 triplets and two singlets (all states assigned to the elastic channel plus Bands 1 and 2 of Ref. 15), and 13ch-sep for the ground state plus 7 triplets and 5 singlets (all states assigned to the elastic channel plus Bands 1, 2, and 3 of Ref. 15). Other acronyms used in this paper are $21 \mathrm{ch}$-sep, $23 \mathrm{ch}-\mathrm{sep}, 28 \mathrm{ch}$ sep, and 33ch-sep, meaning that all states of the MOBSCI calculation up to $10 \mathrm{eV}, 15 \mathrm{eV}, 20 \mathrm{eV}$, and $24 \mathrm{eV}$, respectively, are energetically accessible, and as a consequence, they are included in the sum appearing in the projector of Eq. (3). A summary of the theoretical spectrum is given in Table I. Note that the 33ch-sep approximation has all the MOBSCI channels (real and pseudostates) as electronically open channels. The ionization, $B$, and kinetic, $U$, orbital energies necessary to obtain the BEB cross section were obtained in a HartreeFock calculation with the $6-311++\mathrm{G}(2 \mathrm{~d}, 1 \mathrm{p})$ basis set using the program GAMESS. ${ }^{17}$ The geometry employed in this calculation was optimized at the MP2 level with the same basis set. The calculated ionization potential of phenol is $8.61 \mathrm{eV}$ which agrees well with the experimental value of $8.51 \mathrm{eV}^{19}$ and the value observed in a recent electron impact ionization investigation. ${ }^{20}$

\section{ATOMIC OPTICAL MODEL (OM)}

The independent atom model with screening corrected additivity rule (IAM-SCAR) method has had significant use in calculating electron scattering cross sections for a wide variety of molecular targets (see, e.g., Refs. 21-26) and over a broad energy $\left(E_{0}\right)$ range (typically $\sim 1-5000 \mathrm{eV}$ ). As a consequence, we only précis the key points of that approach here.

The first subjects of our computations are the individual atoms that form phenol, namely, carbon (C), oxygen (O), and hydrogen $(\mathrm{H})$. The atomic $\mathrm{OM}$ is based on a potential scattering approach, where the local complex potential $V(r)$ is given by

$$
V(r)=V_{\mathrm{s}}(r)+V_{\mathrm{ex}}(r)+V_{\mathrm{p}}(r)+i V_{\mathrm{a}}(r) .
$$

In the above equation $V_{\mathrm{s}}(r)$ is the standard Hartree potential of the target, $V_{\mathrm{ex}}(r)$ represents the exchange interaction of Riley and Truhlar, ${ }^{27} V_{\mathrm{p}}(r)$ is the polarisation potential of Zhang et al. ${ }^{28}$ and $V_{\mathrm{a}}(r)$ is the imaginary absorption potential of Staszewska et al. ${ }^{29}$ Due to the last term in Eq. (1), the OM potential approach yields a complex phase shift. This allows for the calculation of the DCSs and ICSs for elastic and inelastic (taken to mean all excited electronic states and ionisation combined together) scattering, as well as the TCS as the sum of those ICSs for each atom.

TABLE I. Summary of the calculated and experimental excitation energies below $7 \mathrm{eV}$ for phenol from Ref. 15 . The MOBSCI calculation aimed to reproduce all 12 states of the FSCI calculation lying below $7 \mathrm{eV}$. These are 7 triplet and 5 singlet states listed below. The remaining MOBSCI pseudostates are 11 singlet states opening up at $8.31,8.83,8.89,9.37,9.45,14.43,17.55,19.15,20.34,21.35$, and $23.82 \mathrm{eV}$ and 9 triplet states at $8.25,8.37,8.79$, $12.65,16.01,18.46,19.75,21.10$, and $23.26 \mathrm{eV}$.

\begin{tabular}{ccccccc}
\hline \hline & & \multicolumn{3}{c}{$\begin{array}{c}\text { Dipole } \\
\text { transition }\end{array}$} \\
\cline { 5 - 7 } & Expt. band & Symmetry & $(\mathrm{D})$ & FSCI & MOBSCI & TD-DFT \\
\hline TRIPLET & Band 1 (3.4-4.3 eV) & $A^{\prime}$ & $\ldots$ & 3.29 & 3.57 & 3.71 \\
& Band 1 (3.4-4.3 eV) & $A^{\prime}$ & $\ldots$ & 4.49 & 4.73 & 4.10 \\
& Band 2 (4.3-5.4 eV) & $A^{\prime}$ & $\ldots$ & 4.78 & 4.90 & 4.53 \\
& Band 2 (4.3-5.4 eV) & $A^{\prime \prime}$ & $\ldots$ & 5.94 & 6.03 & 5.06 \\
& Band 2 (4.3-5.4 eV) & $A^{\prime}$ & $\ldots$ & 5.73 & 6.16 & 5.30 \\
& Band 3 (5.4-6.3 eV) & $A^{\prime \prime}$ & $\ldots$ & 6.53 & 6.78 & 5.53 \\
& Band 3 (5.4-6.3 eV) & $A^{\prime \prime}$ & $\ldots$ & 6.86 & 6.99 & 5.90 \\
& Band 2 (4.3-5.4 eV) & $A^{\prime}$ & 1.04 & 5.82 & 6.09 & 4.99 \\
& Band 2 (4.3-5.4 eV) & $A^{\prime \prime}$ & 0.08 & 6.06 & 6.21 & 5.13 \\
& Band 3 (5.4-6.3 eV) & $A^{\prime}$ & 0.35 & 6.12 & 6.80 & 5.76 \\
& Band 3 (5.4-6.3 eV) & $A^{\prime \prime}$ & 1.03 & 6.68 & 6.86 & 5.57 \\
& Band 3 (5.4-6.3 eV) & $A^{\prime \prime}$ & 0.03 & 6.86 & 6.99 & 5.98 \\
\hline \hline
\end{tabular}




\section{SCREENING CORRECTED ADDITIVITY RULE (SCAR) AND ROTATIONAL EXCITATIONS}

We subsequently calculate the electron- $\mathrm{C}_{6} \mathrm{H}_{5} \mathrm{OH}$ cross sections by applying the additivity rule (AR) to the OM results of each atom. In that approach, the molecular scattering amplitude stems from the sum of all the relevant atomic amplitudes, including the phase coefficients, which gives the elastic DCSs for phenol. Elastic ICSs can then be determined by integrating those DCSs. The sum of the elastic and absorption ICSs (for all inelastic processes except rotations and vibrations) then gives the molecular TCS. However, the AR does not account for the target molecular structure, so that it is only applicable when the incident electrons are so fast that they effectively "see" the target molecule as a sum of the individual atoms (typically above $\sim 100 \mathrm{eV}$ ). To try and overcome this limitation, Blanco and García ${ }^{30,31}$ introduced the SCAR method which employs some screening coefficients to account for the geometry of the molecule (atomic positions and bond lengths). The IAMSCAR method as described does not account for rotational and vibrational excitations. However, for polar molecules such as $\mathrm{C}_{6} \mathrm{H}_{5} \mathrm{OH}$, additional dipole-induced excitation cross sections can be computed using the approach of Jain. ${ }^{32}$ In that method, rotational excitation DCSs and ICSs for a free electric dipole are calculated within the framework of the first Born approximation. Those results can now be incorporated into our IAM-SCAR results in an incoherent way, just by adding up the cross sections as independent channels. We call the cross sections that result from this latter process as "IAM-SCAR + rotations."

There is a body of evidence that suggests that the IAM-SCAR approach for non-polar molecules and IAMSCAR + rotations method for polar species ${ }^{21-26}$ provides a good description of the measured cross sections down to $E_{0}=20 \mathrm{eV}$. However, there are also some examples ${ }^{33-35}$ where the comparison between these two approaches and the measured cross sections is only reasonable at energies above $\sim 50 \mathrm{eV}$. Therefore, one of the rationales of the present investigation was to test the efficacy of the IAM-SCAR plus rotations results against corresponding results from our fully $a b$ initio SMCPP at the $N_{\text {open }}$ ch-sep level computations, at representative energies of $50 \mathrm{eV}$ and below.

\section{EXPERIMENTAL APPARATUS FOR THE TOTAL CROSS SECTIONS: DETAILS AND METHOD}

The present total cross sections were measured with an apparatus, located in Madrid, whose functionality is based on a magnetic confinement of an electron beam when it enters into the collision chamber until its ultimate detection. The role of the main magnetic field $(0.2 \mathrm{~T})$ is simply to translate the electrons, which exhibit the precise angle and energy that resulted from a potential collision, along the central axis to the end of the collision chamber. This apparatus has already been recently described in detail, ${ }^{21,22}$ so that only a brief summary of its more important points is given here.

The apparatus consists of three regions: (i) electron gun region, (ii) collision chamber region, and (iii) the analyzer-detector region. Each region is connected by small orifices and each has independent magnetic fields. Electrons are generated by thermionic emission from a tungsten filament and accelerated to a kinetic energy $E_{0}$ before passing into the collision chamber. The magnetic field of the electron gun region $\left(B_{\mathrm{g}}\right)$, oriented opposing the main field $B$, ensures a low angular divergence of this electron beam by locally compensating for $B$ and preventing electrons leaving the filament in oblique directions to pass through the collimators. The collision chamber has a geometrical length of $140 \mathrm{~mm}$ and so is sufficiently large compared to the limiting apertures (of $1 \mathrm{~mm}$ and $2.3 \mathrm{~mm}$ diameter) to ensure a well-defined region of constant pressure. Phenol is introduced into the system using a heated variable leak valve, from a steel sample-container maintained at around $50^{\circ} \mathrm{C}$ by means of various silicone heater mats. The chamber wall in principle can partly absorb the heat dissipated by the magnetic coils, depending on the pumping speed of the cooling liquid through the chamber's water jacket. ${ }^{21,22}$ Hence, the balance between solenoid current and water speed can be utilized in order to maintain a convenient chamber temperature and avoid condensation of the phenol on the inner chamber walls. In this application, the collision cell temperature was held at the same temperature as the samplecontainer to ensure the phenol pressure was maintained at a constant value. The pressure in the scattering chamber (or cell) is determined by an absolute capacitance manometer (MKS Baratron 627B, Germany), while the temperature is measured (and monitored) using a K-type thermocouple in thermal contact with the inner chamber wall. Due to the magnetic confinement, the effective localization of the electrons after scattering (but before entering the analyzer/detector region) is within $1 \mathrm{~mm}$ around the central axis. After traversing the collision chamber, electrons are selected by a retarding potential analyzer (RPA) so that only those with parallel (axial) components of kinetic energy $\geqslant e V_{\mathrm{R}}$ (where $e=1.6 \times 10^{-19} \mathrm{C}$ and $V_{\mathrm{R}}=$ retarding potential) continue towards the detector. The detector assembly is formed by two micro-channel plates and an anode, and is typically polarized with a voltage drop of around $+2 \mathrm{kV}$. It is operated in a single-pulse counting mode and connected, via some additional electronics, to a computer running a custom LabView (National Instruments) program which registers and analyses the signal.

Phenol, with a stated purity better than $99 \%$, is a white solid at room temperature but readily sublimes under vacuum. In this case, our sample was purchased from Sigma-Aldrich and it was further purified through the performance of freeze-pump-thaw cycles. Before each experiment, the energy resolution defined as $\Delta E=e\left(V_{\mathrm{R}, 90}-V_{\mathrm{R}, 10}\right) / 2$, with $V_{\mathrm{R}, 90}$ and $V_{\mathrm{R}, 10}$ being the retarding potentials leading to $90 \%$ and $10 \%$ of the transmitted electrons, respectively, was determined from the transmission curve $I\left(V_{\mathrm{R}}\right)$ in vacuum. Here, $I$ is the transmitted beam intensity (electron count rate). This energy resolution was usually found to be almost identical to the fullwidth-at-half-maximum (FWHM) of the derivative of $I\left(V_{R}\right)$ and is given, at each energy, in Table II. Subsequently, the retarding potential was fixed to define the cut-off energy ${ }^{21,22}$ and then the transmitted intensity as a function of the gas pressure was recorded. In the present study, the pressure range was 0.2-4 mTorr, depending on the energy, but always ensuring the attenuation was less then $50 \%$ of the incident 
TABLE II. Measured total cross sections $\left(\sigma_{\text {exp }}\right)$, the correction to the TCSs due to the forward angle scattering effect $\left(\sigma_{\text {forw }}\right)$ and the "true" TCSs $(\sigma)$, as a function of energy, for electron collisions with phenol, in $10^{-16} \mathrm{~cm}^{2}$ units. For each energy, the reproducibility index, energy resolution $(\Delta E)$ and angular discrimination $(\Delta \theta)$ are also given.

\begin{tabular}{|c|c|c|c|c|c|c|}
\hline$E_{0}(\mathrm{eV})$ & $\sigma_{\exp }\left(10^{-16} \mathrm{~cm}^{2}\right)$ & $\begin{array}{l}\text { Reproducibility } \\
\text { (\%) }\end{array}$ & $\Delta E(\mathrm{eV})$ & $\begin{array}{l}\Delta \theta \\
\left({ }^{\circ}\right)\end{array}$ & $\sigma_{\text {forw }}\left(10^{-16} \mathrm{~cm}^{2}\right)$ & $\sigma\left(10^{-16} \mathrm{~cm}^{2}\right)$ \\
\hline 10 & 34.8 & 12 & 3.5 & 36.3 & 18.0 & 52.8 \\
\hline 15 & 36.8 & 7 & 1.8 & 20.3 & 12.3 & 49.1 \\
\hline 20 & 30.6 & 8 & 2.2 & 19.4 & 11.5 & 42.1 \\
\hline 30 & 30.1 & 5 & 2.0 & 15.0 & 7.72 & 37.8 \\
\hline 40 & 31.2 & 8 & 2.3 & 13.9 & 6.95 & 38.2 \\
\hline 50 & 29.9 & 12 & 1.8 & 10.9 & 5.43 & 35.2 \\
\hline 70 & 25.1 & 8 & 2.8 & 11.5 & 4.88 & 30.0 \\
\hline 100 & 23.7 & 2 & 2.2 & 8.5 & 4.15 & 27.9 \\
\hline 150 & 18.5 & 6 & 2.0 & 6.6 & 3.12 & 21.6 \\
\hline 200 & 18.1 & 6 & 2.2 & 6.0 & 2.58 & 20.7 \\
\hline 300 & 14.5 & 6 & 1.9 & 4.6 & 2.04 & 16.5 \\
\hline 500 & 9.7 & 6 & 2.7 & 4.2 & 1.51 & 11.2 \\
\hline 700 & 8.7 & 4 & 2.3 & 3.3 & 1.24 & 9.9 \\
\hline 1000 & 5.9 & 3 & 2.4 & 2.8 & 0.97 & 6.9 \\
\hline
\end{tabular}

intensity in order to avoid multiple scattering effects. For each incident electron energy in the range of $10-1000 \mathrm{eV}$ (see Table II), a series of 7-10 attenuation curves, each normally comprising 7-12 data pairs ( $I$ and pressure $(p)$ ), were then acquired. Those data points were now fitted with an exponential curve $I(p)$, from which the experimental TCS, $\sigma_{\text {exp }}$, is obtained according to the Beer-Lambert law

$$
I=I_{0} e^{-n l \sigma_{\exp }}=I_{0} e^{-p l \sigma_{\exp } / k T} .
$$

Note that in Eq. (12), $I_{0}$ is the intensity of the in vacuum non-attenuated beam, $n$ is the number density of the phenol gas, $l=0.1413 \mathrm{~m}$ is the effective collision chamber length, ${ }^{21} k$ is Boltzmann's constant, and $T=\sqrt{T_{\mathrm{c}} T_{\mathrm{m}}}$ is the gas temperature (K) calculated according to the thermal transpiration effect ${ }^{36}$ between the manometer at $T_{\mathrm{m}}$ and the collision chamber at $T_{\mathrm{c}}$.

The experimental uncertainties on our TCSs are no worse than $\sim 10 \%$, including contributions due to uncertainties in the collision chamber length, the phenol gas pressure (the major contributor), the temperature, and the true incident beam energy. Furthermore a "reproducibility index," reflecting the standard deviation between sets of $I(p)$ versus $p$ curves from the same series, in the range of $2 \%-12 \%$ was also found (see Table II for the exact value at each $E_{0}$ ). The "reproducibility index" comprised contributions from the filament emission stability, temperature stability, (electronic) signal fluctuations, and an uncertainty associated with the fitting to the attenuation curves. Combining the aforementioned factors, one obtains a general precision in the TCS determination in the range of $8 \%-16 \%$, depending on the energy, for our $10-1000 \mathrm{eV}$ investigation.

In addition to this largely statistical uncertainty, the angular acceptance $\Delta \theta$ of the apparatus is a limiting aspect and represents a potentially important source of systematic error. Here, elastically or rotationally and vibrationally inelastically scattered electrons at small angles $\left(0^{\circ} \leqslant \theta \leqslant \Delta \theta^{\circ}\right)$ or near $180^{\circ}\left(180^{\circ}-\Delta \theta^{\circ} \leqslant \theta \leqslant 180^{\circ}\right)$, for the case of backscattered electrons, are not distinguished from the unscattered electrons and so will not be accounted for in our determination of the measured TCS $\left(\sigma_{\exp }\right)$. Under these circumstances, $\sigma_{\exp }$ will always be lower (and sometimes significantly lower ${ }^{21}$ ) than the "true" value of the TCS $(\sigma)$. The angular discrimination in the forward direction $(\Delta \theta)$ can be determined from the energy resolution $\Delta E$ via $^{21,22}$

$$
\Delta \theta=\sin ^{-1}\left(\sqrt{\Delta E / E_{0}}\right) .
$$

The values of $\Delta \theta$ for the present measurements were found to lie between $3^{\circ}$ and $36^{\circ}$, and are listed in Table II. This means that any valid comparison to our SMCPP + BEB and IAMSCAR + rotations theory TCSs needs to take into account that the present measured values $\sigma_{\text {exp }}$ actually represent only partial values

$$
\sigma_{\exp }(E) \approx \sigma(E)-\sigma_{\text {forw }}(E) .
$$

Our procedure for calculating $\sigma_{\text {forw }}(E)$, using the IAMSCAR elastic DCS and the Born-based rotational DCS, the vibrational scattering cross sections being ignored as they are expected to be relatively much smaller in magnitude in our $10-1000 \mathrm{eV}$ energy range, ${ }^{37}$ can be found in Fuss et al. ${ }^{21}$ and Sanz et $a .^{22}$ and so we do not repeat that detail here. Rather, we simply note that our values for $\sigma_{\text {forw }}$ and $\sigma$ can be found in Table II. We reiterate only a comparison of $\sigma(E)$ with corresponding theory is meaningful and we discuss this in more detail shortly in our relevant results and discussion section.

\section{RESULTS AND DISCUSSION: ELECTRONIC MULTICHANNEL EFFECTS ON THE ELASTIC ELECTRON-PHENOL DIFFERENTIAL CROSS SECTIONS}

Figures 1-3 show the $\ell_{\max }$ convergence for our calculated differential cross sections for a selection of phenol's excited electronic states, obtained with the scattering amplitude of Eq. (6), with respect to the converged result obtained from 

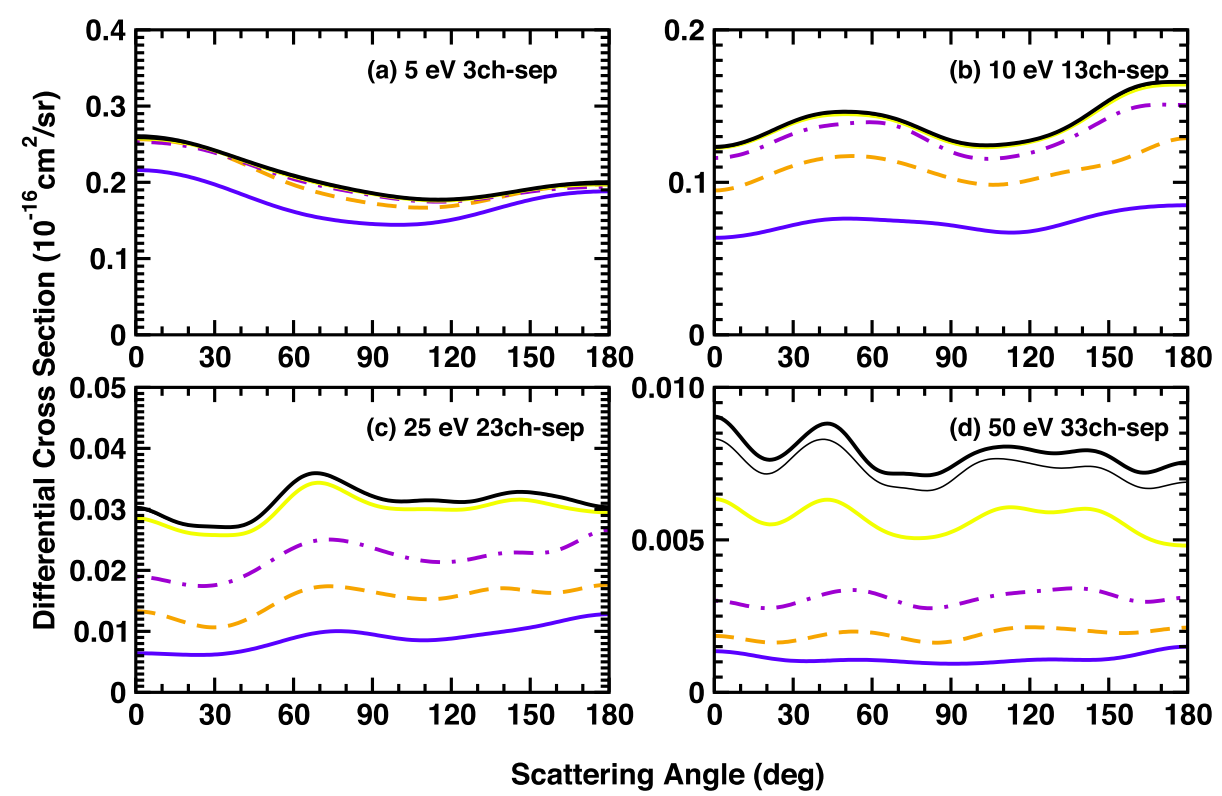

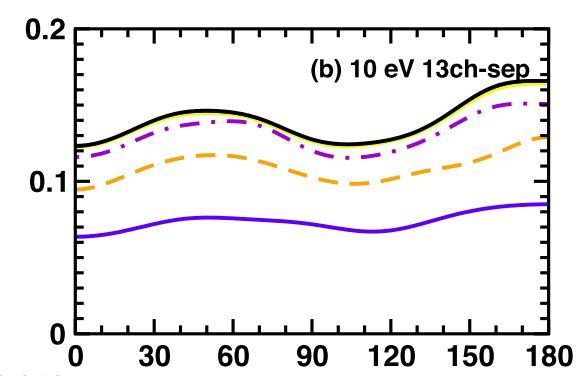

FIG. 1. Differential cross sections obtained from the scattering amplitude given by Eq. (6) for the excitation of the first triplet state of phenol at (a) $5.0 \mathrm{eV}$ at a $3 \mathrm{ch}$-sep approximation, (b) $10 \mathrm{eV}$ at a 13ch-sep approximation, (c) $25 \mathrm{eV}$ at a $23 \mathrm{ch}$-sep approximation, and (d) $50 \mathrm{eV}$ at a 33ch-sep approximation. Full (blue) line: $\ell_{\max }=4$; long dashed (orange) line: $\ell_{\max }=5$; dashed-dotted (violet) line: $\ell_{\max }=6$; full (yellow) line: $\ell_{\max }=8$; thin full (black) line: $\ell_{\max }$ $=10$; thick full (black) line: converged result from Eq. (5) with $\ell_{\text {max }}=13$.
Eq. (5), with $\ell_{\max }=13$. We have performed this study for one excited state from each experimental band (1st triplet state of phenol that belongs to Band 1, 1st singlet of phenol that belongs to Band 2, and 4th singlet state of phenol that belongs to Band 3) of the experimentally resolved bands in the energy loss spectra of Ref. 15. The electronic excitation cross sections of those bands are the subject of study of an accompanying paper $^{5}$ in this same journal issue. Figure 1 shows the DCSs for 5, 10, 25, and $50 \mathrm{eV}$ for the 1st triplet of phenol calculated at the $3 \mathrm{ch}$-sep, 13ch-sep, 23ch-sep, and $33 \mathrm{ch}$-sep levels of approximation, respectively. In Figure 2, we present our DCSs for $8,15,25$, and $50 \mathrm{eV}$ for the $1 \mathrm{st}$ singlet of phenol calculated at the 8ch-sep, 13ch-sep, 23chsep, and 33ch-sep levels of approximation, respectively. Next, in Figure 3, we show DCSs for 8, 15, 25, and $50 \mathrm{eV}$ for the 4th singlet of phenol (chosen because it has the largest dipole transition of Band $3^{15}$ ) calculated at the 8ch-sep, 23ch-sep, $28 \mathrm{ch}$-sep, and $33 \mathrm{ch}$-sep levels of approximation, respectively.
The results of these 3 figures clearly show that $\ell_{\max }=4$ is not enough for convergence, even for an impact energy as low as $5 \mathrm{eV}$ or $8 \mathrm{eV}$ for the first triplet or first singlet, respectively. For a reasonable convergence, $\ell_{\max }=8$ is necessary for energies up to $25 \mathrm{eV}$, and for higher energies, $\ell_{\max }=10$ or 13 is required. For $50 \mathrm{eV}$, the DCS obtained with the scattering amplitude from Eq. (6) with $\ell_{\max }=4$ can be less than $10 \%$ of the converged DCS. This is clearly unsatisfactory. For justifying the use of $\ell_{\text {max }}=4$ in Eq. (6), we would need an indication that the higher partial wave contributions from the SMCPP method are less significant than simply zeroing them out (for the triplet transitions) or less significant than replacing them with a 1st Born approximation for the higher partial waves (for singlet transitions with the Born closure). Unfortunately, as will be seen in the elastic study ahead, there is no indication that we could cut $\ell_{\max }$ to a value as low as 4 .

Figures 4-6 show the multichannel effects on the 1st triplet state, and on the selected ${ }^{15}$ Band 2 of phenol for impact
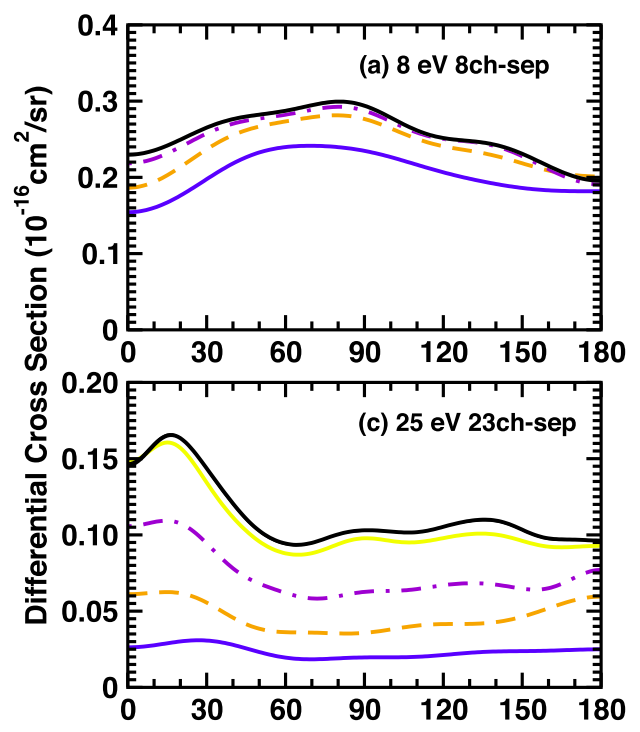
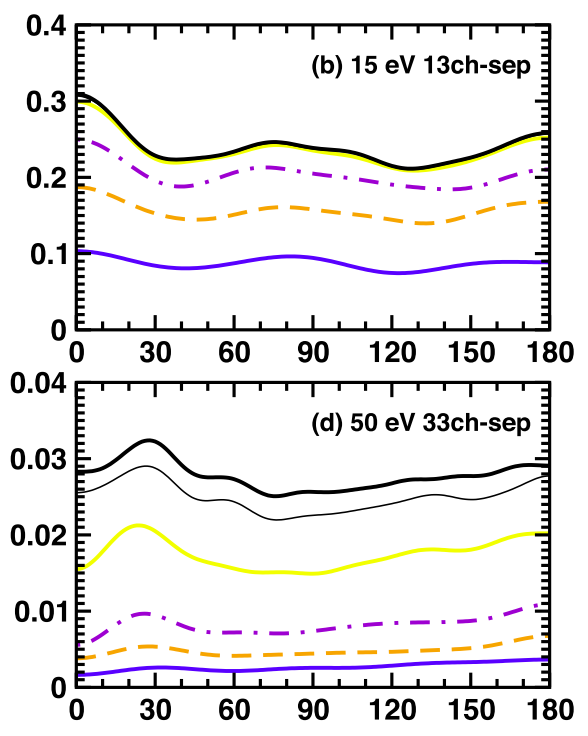

FIG. 2. Differential cross sections obtained from the scattering amplitude given by Eq. (6) (no closure) for the excitation of the first singlet state of phenol. Labels for $\ell_{\max }$ are the same as those used in Fig. 1, but at (a), 8chsep level of approximation for $8 \mathrm{eV}$, (b) $13 \mathrm{ch}$-sep for $15 \mathrm{eV}$, (c) $23 \mathrm{ch}$-sep for $25 \mathrm{eV}$, and (d) $33 \mathrm{ch}$-sep for $50 \mathrm{eV}$ electron impact energy. 

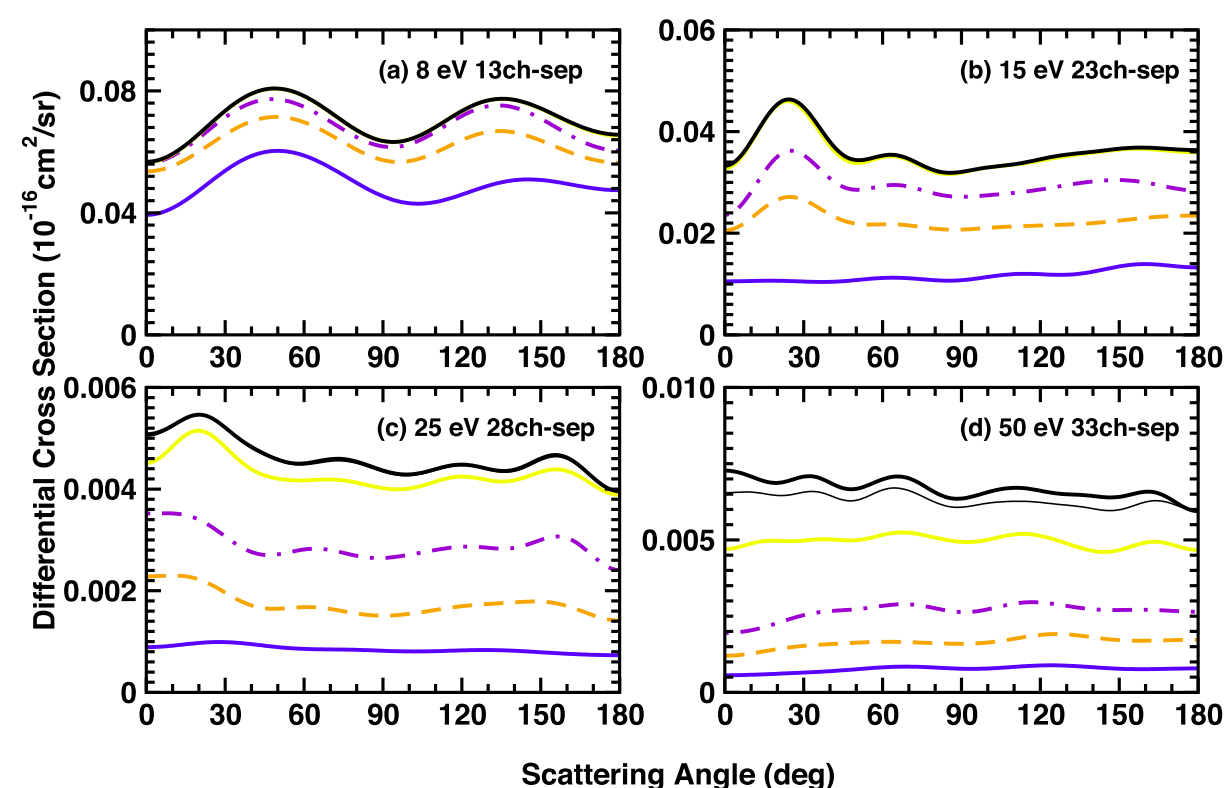

FIG. 3. Differential cross sections obtained from the scattering amplitude given by Eq. (6) (no closure) for the excitation of the fourth singlet state of phenol. Labels for $\ell_{\max }$ are the same as those used in Fig. 1, but at (a), 13chsep level of approximation for $8 \mathrm{eV}$, (b) 23ch-sep for $15 \mathrm{eV}$, (c) 28ch-sep for $25 \mathrm{eV}$, and (d) 33ch-sep for $50 \mathrm{eV}$ electron impact energy. energies varying from 8 to $50 \mathrm{eV}$. In particular, Figure 4 shows these effects on the electronic excitation DCSs of the ${ }^{3} A^{\prime}$ state of phenol (see Table I) for $8,10,12$, and $15 \mathrm{eV}$, at the $3 \mathrm{ch}$-sep, 8ch-sep, 13ch-sep, 21ch-sep, and 23ch-sep levels. The level of coupling is defined by the electronic states that are energetically open according to the impact energy and thresholds given in Table I. Figure 5 shows the multichannel influence at the higher impact energies, 20, 30, 40, and $50 \mathrm{eV}$ for this transition. The channel couplings shown in this figure are at the 3ch-sep, 8ch-sep, 13ch-sep, 23ch-sep, 28ch-sep, and the 33ch-sep levels of approximation. In accord with our previous study with ethylene, ${ }^{4}$ we see for phenol that by increasing the number of channels, the probability flux to a particular state $\left({ }^{3} A^{\prime}\right.$ in this case) decreases as we open more electronic states, especially at the higher energies. About the same trend is also seen in Fig. 6 for the electronic excitation of the experimental Band 2, formed by 3 triplets and 2 singlets (see Table I), for electron impact energy ranging again from 8 to $50 \mathrm{eV}$. In general, the results follow the trend of decreasing the flux into the relevant band excitation as we open more channels. In this case, however, there are a few exceptions, e.g., at $15 \mathrm{eV}$ electron impact energy, the 13ch-sep DCSs appear stronger in magnitude than the 8ch-sep results and there is also an inversion between the $28 \mathrm{ch}$-sep and $33 \mathrm{ch}$-sep DCSs for 30 and $50 \mathrm{eV}$. Nonetheless, we reiterate that the overall trend we have highlighted typically holds for the states incorporated in Band 2. Further discussion on the electronic excitation of phenol is carried out in an accompanying paper 5 also published in this journal issue.

Figures 7 and 8 show elastic DCSs and the influence of multichannel effects in this transition. As in the inelastic case, the DCSs decrease their magnitude as we increase the number of open channels. We compare our elastic phenol differential cross sections with the experimental data obtained by Cho et al. ${ }^{38}$ for benzene, a molecule differing from phenol by the replacement of a $\mathrm{H}$ atom in benzene with a Hydroxyl

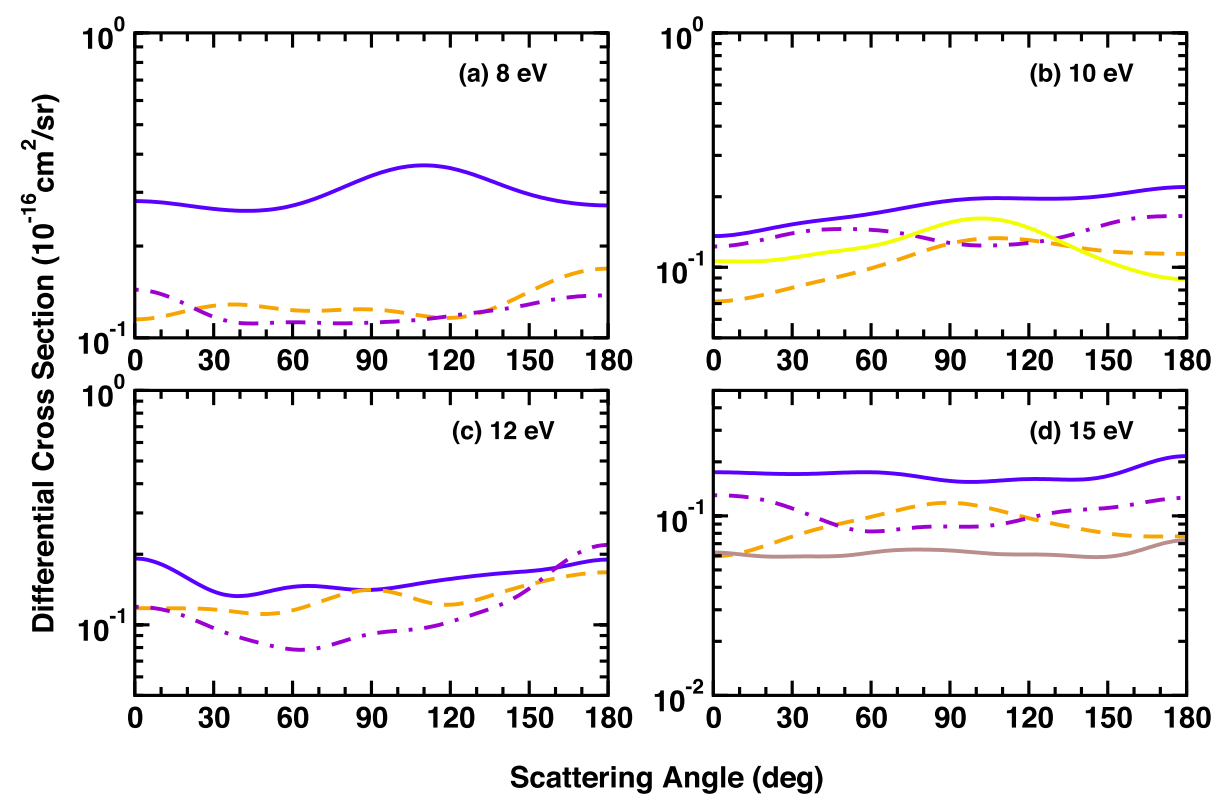

FIG. 4. Multichannel coupling influence on the differential cross sections for the excitation of the first triplet state of phenol at (a) $8.0 \mathrm{eV}$, (b) $10.0 \mathrm{eV}$, (c) $12.0 \mathrm{eV}$, and (d) $15.0 \mathrm{eV}$. Full (blue) line: 3ch-sep; dashed (orange) line: $8 \mathrm{ch}$-sep; dashed-dotted (violet) line: 13ch-sep; full (yellow) line: 21ch-sep; full (brown) line: 23ch-sep. 

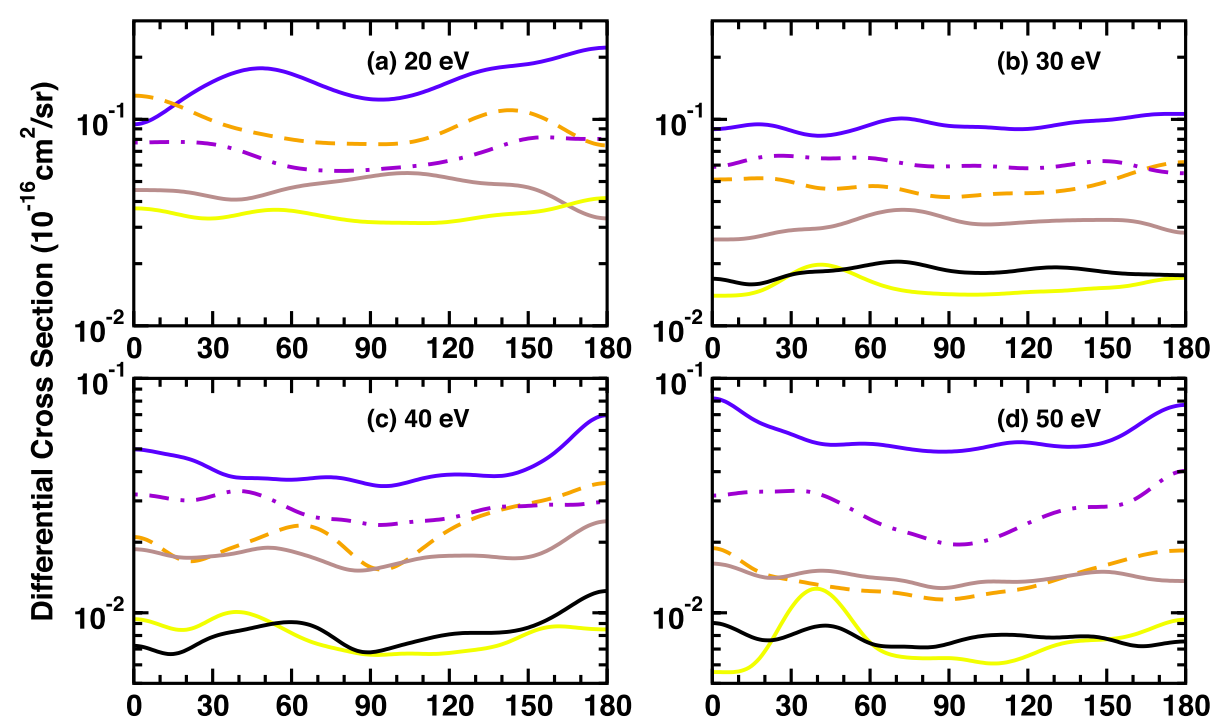

Scattering Angle (deg)

$(\mathrm{OH})$ group. Benzene has no dipole moment, but as a result of this replacement, phenol molecules have a permanent dipole moment. The dipole moment in phenol is not too large $(66 \%$ of the water molecule dipole) and affects mainly the scattering cross sections in the more forward direction. Figure 7 shows elastic DCSs for impact energies of 5, 8, 10, and $12 \mathrm{eV}$. It is possible to note from this figure that at $5 \mathrm{eV}$, the multichannel effect seems to be not too important. On the other hand, for higher energies, the presence of multichannel effects changes substantially the DCS when compared to the one obtained at a $1 \mathrm{ch}$-sep level of approximation. At $8 \mathrm{eV}$, the $3 \mathrm{ch}$-sep, 8ch-sep, and 13ch-sep elastic cross sections are in better agreement with the experiments than the usual sep approximation which usually has only the elastic channel as open (here, named 1chsep). To our surprise, at $10 \mathrm{eV}$, the $13 \mathrm{ch}$-sep approximation gave worse results than the $3 \mathrm{ch}$-sep and $8 \mathrm{ch}$-sep calculations when compared with the experimental data for benzene. However, as we further increase the number of channels to a 21ch-sep level of approximation, the DCS for $10 \mathrm{eV}$ increases in size and gets in much better agreement with the experiments. The nature of this instability for some of the $N_{\text {open }}$ ch-sep approximations will be discussed in Sec. VIII. Figure 8 shows the present calculated elastic DCSs for impact energies at 15, 20,30 , and $40 \mathrm{eV}$. The agreement with the experiments is excellent for all energies and, in general, follows the trend that as we open more channels the scattered elastic flux decreases towards the experimental data of benzene. This level of agreement in the benzene and phenol elastic DCSs is somewhat intriguing, as they are different species (although both have ring structures), and we believe might reflect the fact, at least in part, that they both possess very similar dipole polarizabilities. ${ }^{16}$ Such a good agreement invites us to speculate that the inelastic cross section should also match well with our new experiments, which is not the case, as discussed in detail in the accompanying inelastic electronic-state paper. $^{5}$

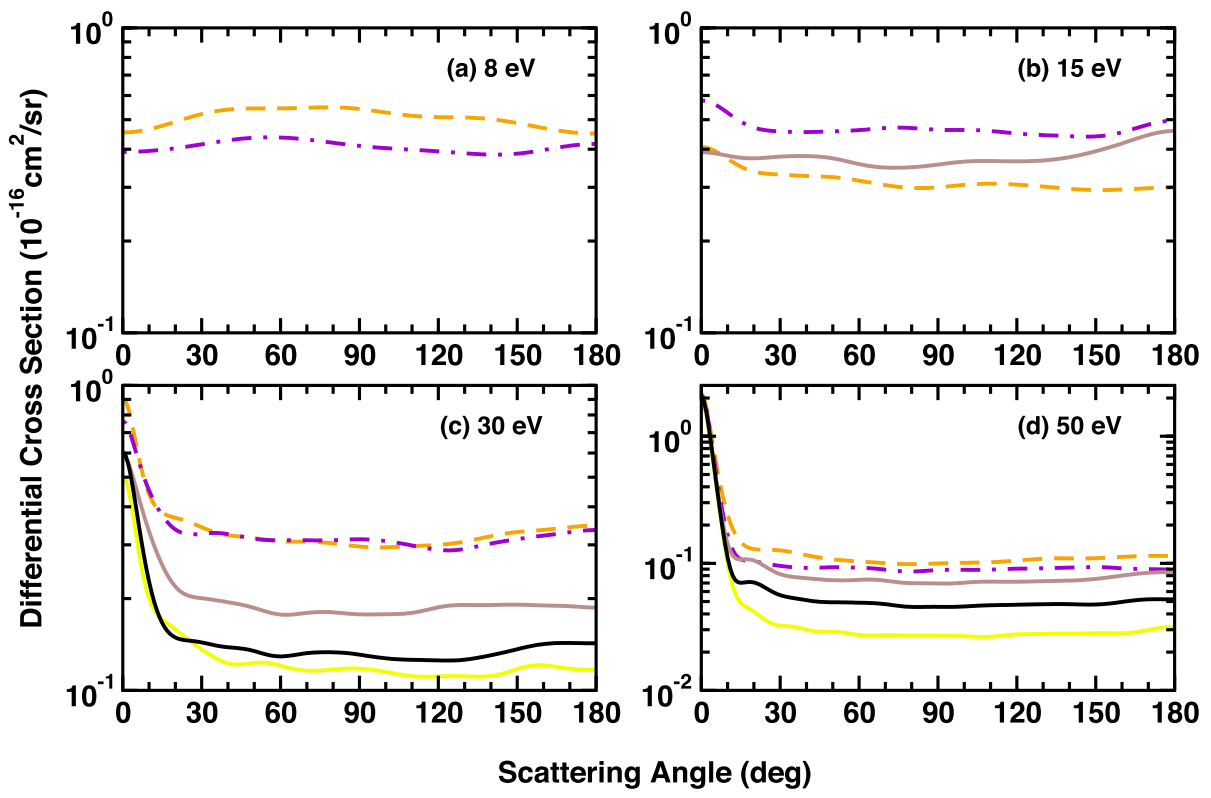

FIG. 6. Multichannel coupling influence on the differential cross sections obtained from the scattering amplitude given by Eq. (7) (with closure, $\ell_{\max }$ $=10$ ) for the excitation of our selected ${ }^{15}$ Band 2 of phenol at (a) $8 \mathrm{eV}$, (b) $15 \mathrm{eV}$, (c) $30 \mathrm{eV}$, and (d) $50 \mathrm{eV}$. Dashed (orange) line: $8 \mathrm{ch}$-sep; dashed-dotted (violet) line: 13ch-sep; full (brown) line: 23ch-sep; full (yellow) line: 28ch-sep; full (black) line: 33ch-sep.

FIG. 5. Multichannel coupling influfor the excitation of the first triplet $30.0 \mathrm{eV}$, (c) $40.0 \mathrm{eV}$, and (d) $50.0 \mathrm{eV}$.
3 , ange) line: $8 \mathrm{ch}$-sep; dashed-dotted (violet) line: 13ch-sep; full (brown) line:
23ch-sep; full (yellow) line: $28 \mathrm{ch}-\mathrm{sep}$; full (black) line: 33ch-sep. 

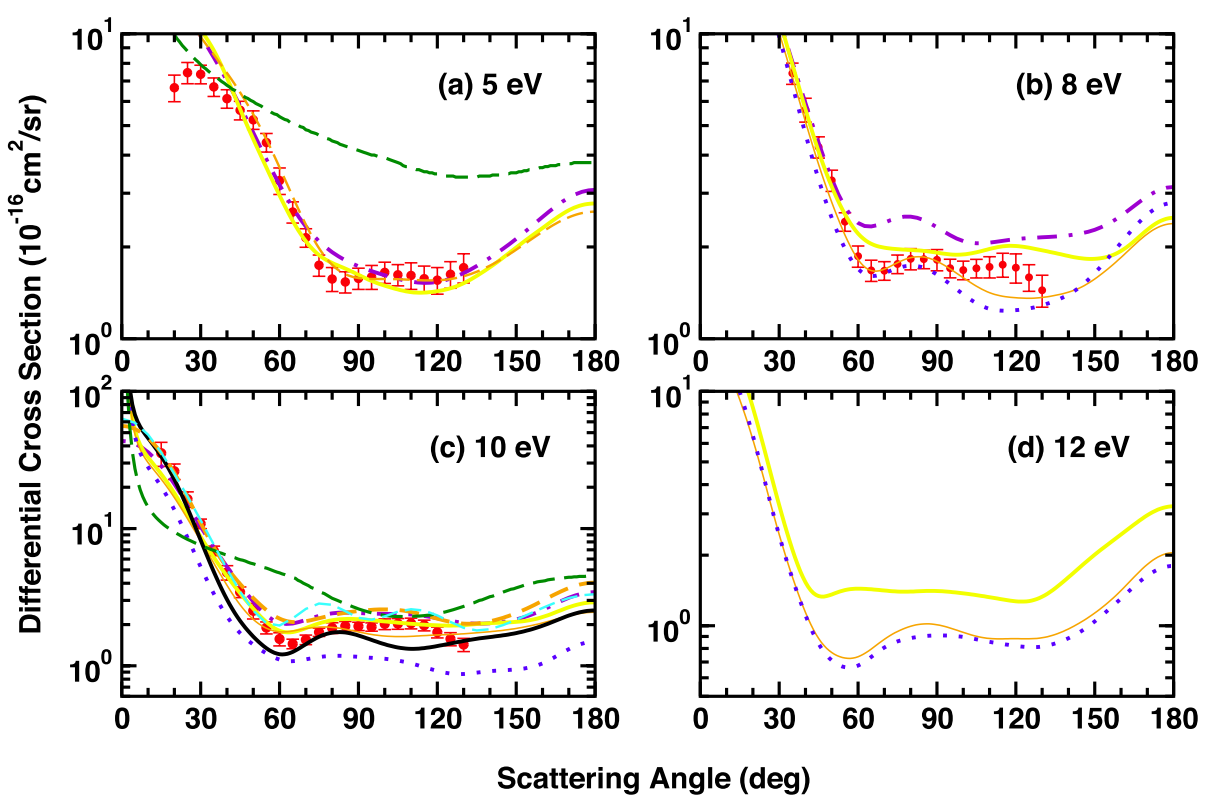

FIG. 7. Multichannel coupling influence on the elastic differential cross sections of phenol for (a) $5.0 \mathrm{eV}$, (b) $8.0 \mathrm{eV}$, (c) $10.0 \mathrm{eV}$, and (d) $12.0 \mathrm{eV}$ electron impact energies. Full (red) circles: experiments of Ref. 38 for benzene; dashed (orange) line: 1ch-sep of Ref. 14; dashed-dotted (violet) line: 1ch-sep; full (yellow) line: 3 ch-sep; thin full (orange) line: $8 \mathrm{ch}$-sep; dotted (blue) line: 13ch-sep; full (black) line: 21ch-sep; thin dashed (green): present IAM-SCAR calculation; dashed (cyan) line: SVIM calculation for benzene of Ref. 39.
In terms of a comparison between our computed IAMSCAR elastic DCS and the measured elastic DCS, we find that the level of overall accord is not as impressive as that just described for the converged SMCPP results. While our IAMSCAR angular distributions do reproduce the gross features of the experimental angular distributions, particularly at the higher impact energies, some disagreement in terms of the absolute magnitude of the cross section are found in Figures 7 and 8. This is not a unique case, the IAM-SCAR approach had, at the lower energies $(<50 \mathrm{eV})$, some difficulties in describing the elastic DCS for the radical $\mathrm{CF}_{3},{ }^{33,34}$ and the bio-molecule 1,4-dioxane ${ }^{35}$ which we believe can be ascribed to the screening corrections not fulfilling their role as well as they might. Nonetheless, for phenol, the IAM-SCAR results are in quite fair agreement with the benzene DCS above about $40 \mathrm{eV}$ energy. A better degree of agreement was found between the elastic DCSs calculated by the SMCPP for phenol and those calculated by the Schwinger variational iterative method (SVIM) for benzene ${ }^{39}$ at 10, 20, 30, and $40 \mathrm{eV}$. The SVIM is a single center expansion method that allows the calculation of cross sections via an optical potential model, which accounts for the contribution of an average number of electronically inelastic processes. Again, we see that the agreement is improved as the impact energy increases.

Figure 9 depicts the angular momentum $\ell_{\max }$ convergence, used in Eq. (5), to obtain the elastic differential cross sections. This figure shows that $\ell_{\max }=4$ can be used only for low energies such as $5 \mathrm{eV}$. As the energy increases, the use of such a low value of angular momentum gives an artificially "wavy" DCS which is smaller than the converged results in magnitude. The good agreement with the measured benzene DCSs, seen in the previous figures, indicates that at least for elastic transitions we should not rely on such small $\ell_{\max }$ values.

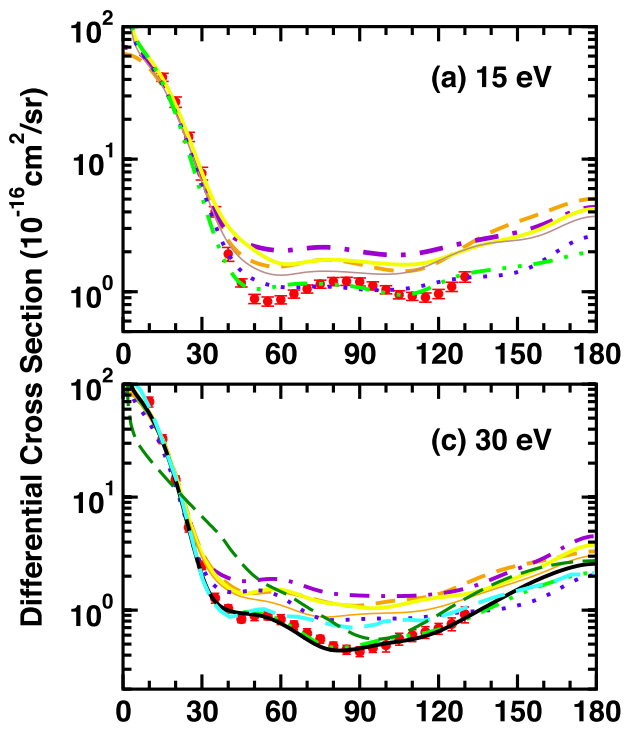

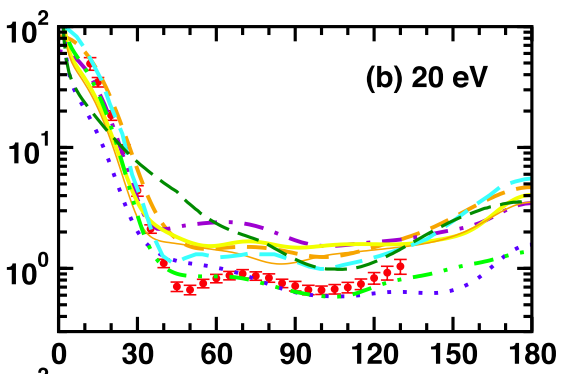

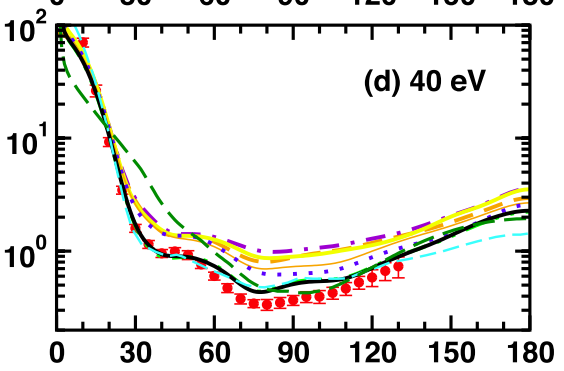

FIG. 8. Multichannel coupling influence on the elastic differential cross sections of phenol for (a) $15.0 \mathrm{eV}$, (b) $20.0 \mathrm{eV}$, (c) $30.0 \mathrm{eV}$, and (d) $40.0 \mathrm{eV}$ electron impact energies. Full (red) circles: experiments of Ref. 38 for benzene; dashed (orange) line: 1chsep of Ref. 14 (unpublished DCS results); dashed-dotted (violet) line: 1chsep; full (yellow) line: 3ch-sep; thin full (orange) line: 8ch-sep; dotted (blue) line: 13ch-sep; dashed-dotted (green) line: 23ch-sep; thick full (black) line: 33ch-sep; thin dashed (green): present IAM-SCAR calculation; thick dashed (cyan) line: SVIM calculation for benzene of Ref. 39. 

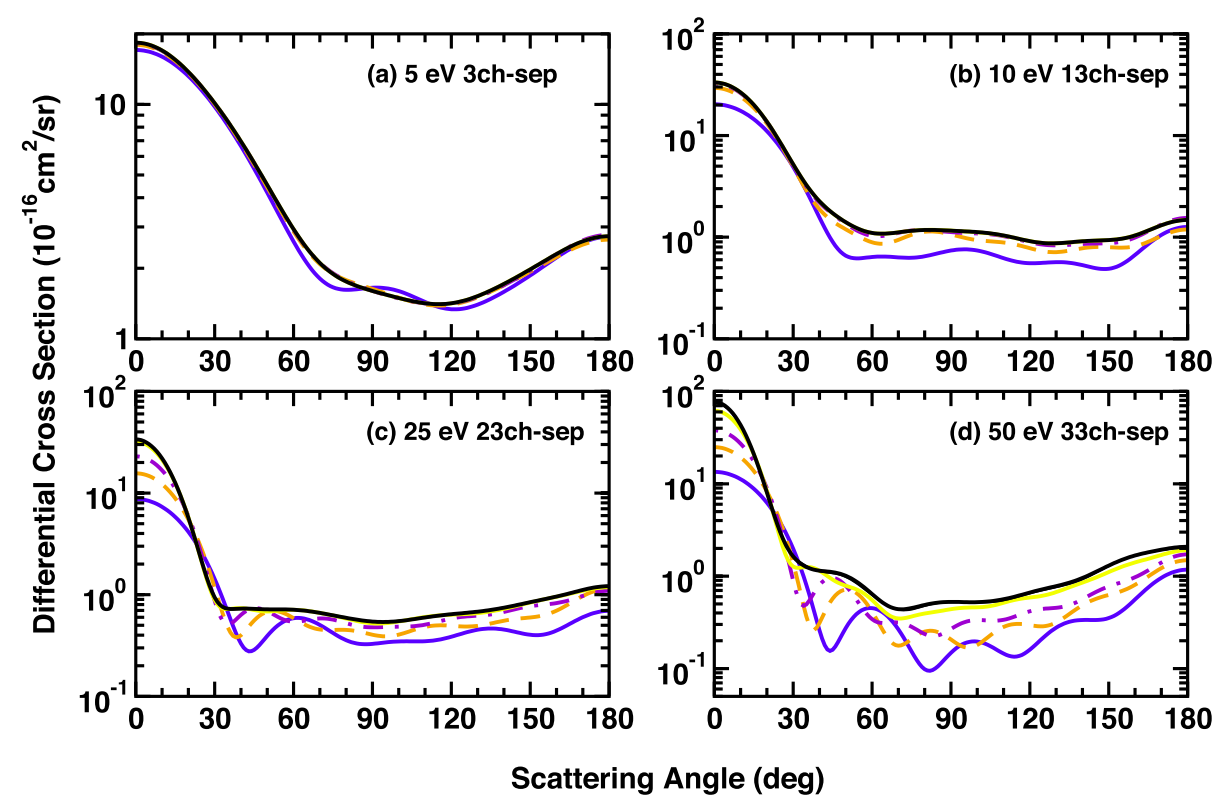

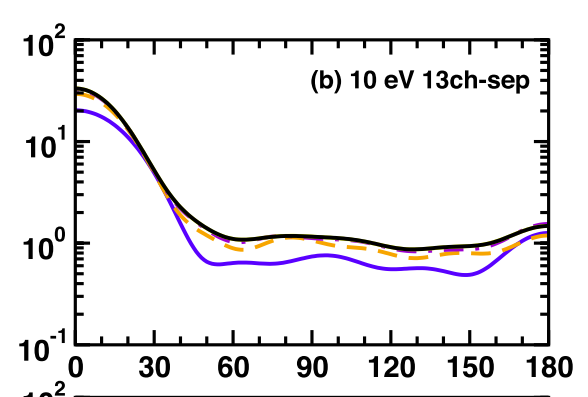

FIG. 9. Differential cross sections for elastic electron scattering by phenol with the same definitions as in Fig. 1.

\section{RESULTS AND DISCUSSION: ELECTRON-PHENOL TOTAL, ELASTIC INTEGRAL, AND ELASTIC MOMENTUM TRANSFER CROSS SECTIONS}

At low energies, only the elastic channel is electronically open and the distortion of the electronic cloud is taken into account by virtual excitations (closed channel space) from the ground state. However, as we increase the impact energy, other electronic states can be excited and if we do not open these channels (i.e., include them in the projector $P$ of Eq. (3) for out-coming flux via the Green function), spurious structures (bumps and troughs) show up in the calculated cross section. These features are more clear in the integral, momentum transfer, and total cross sections, as we will show in the next figures. Due to the presence of $(1-\cos \theta)$ in the angular integration to obtain the MTCS, a permanent dipole moment, even if it is large (resulting in a strong forward peaking DCS), does not contribute as much as in the calculation of the ICS. So, in principle, we would expect to have better agreement between phenol and benzene for the MTCS than for the ICS. However, as noted earlier, the dipole moment of phenol is relatively small and, as we will soon see, the elastic ICS is also comparing well with the corresponding results for benzene. Figure 10 brings a comparison between our calculated momentum transfer cross sections at several levels of approximations (1ch-sep, 3ch-sep, 13ch-sep, 23ch-sep, 28ch-sep, and 33ch-sep) and the experimental measurements of Cho et al. ${ }^{38}$ for benzene. The connected up-triangles are the 1ch-sep calculation. The black curve is a combination of our best channel coupling results for each interval of energy, i.e., 1ch-sep for 1-4 eV, 3ch-sep for around $5 \mathrm{eV}, 13 \mathrm{ch}$-sep for $8-14 \mathrm{eV}, 23 \mathrm{ch}$-sep for around $15 \mathrm{eV}, 28 \mathrm{ch}$-sep for around $20 \mathrm{eV}$, and 33ch-sep for energies above $24.9 \mathrm{eV}$. Here, it is worthwhile noting that the best channel coupling is determined by two parameters: the energy of the incident electron and the thresholds of the excited states obtained according to the MOBSCI strategy. So, for example, for the incident electron energy of $8 \mathrm{eV}$, there are 13-states (the 7 triplets and the 5 singlets displayed in Table I plus the ground state) that became energetically allowed. All other 20 excited states, referred to in the text as pseudostates (for details, please, see the caption of Table I), have their thresholds above $8 \mathrm{eV}$ and were treated as closed channels in this case. Our calculated MTCSs show clearly the trend seen before, i.e., the scattered flux decreases in the elastic channel as a result of opening more electronic channels. The 23ch-sep for 15 and $20 \mathrm{eV}$ (not as much), and the 33ch-sep approximations for 30 and $40 \mathrm{eV}$ are in very reasonable agreement with the experiments. At lower energy, the $13 \mathrm{ch}$-sep approximation gives good results for 8 and $10 \mathrm{eV}$, as we should expect from the comparison of the DCS results. Spikes and troughs are present in this figure, and in order

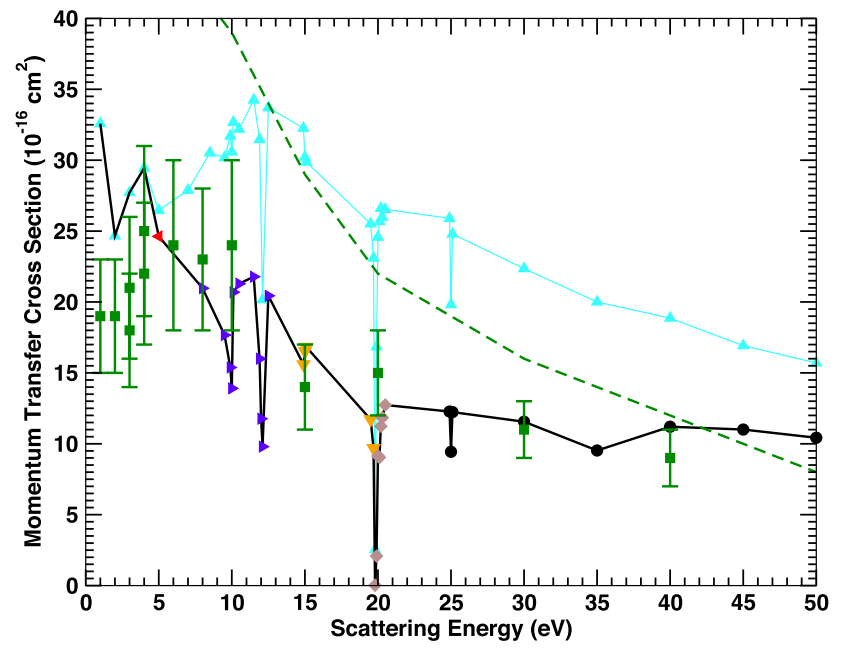

FIG. 10. MTCSs for phenol in the energy region 0-50 eV. Up (cyan) triangles: 1ch-sep; left (red) triangles: $3 \mathrm{ch}$-sep; right (blue) triangles: $13 \mathrm{ch}$ sep; down (orange) triangles: 23ch-sep; (brown) diamonds: 28ch-sep; filled (black) circles: 33ch-sep; full (black) curve: connecting our best SMCPP channel coupling results for each interval of energy (see text); thin dashed (green): present IAM-SCAR calculation; filled (dark green) squares: experimental results of Ref. 38 for benzene. SMCPP results presented in this figure were obtained in a calculation without use of the Born-closure procedure (see Sec. IX for details). 


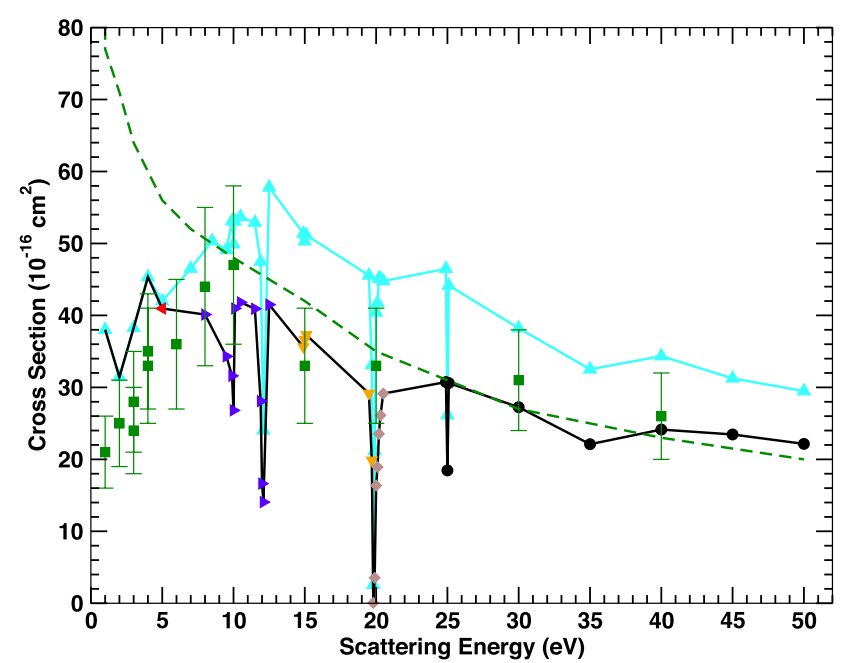

FIG. 11. ICS for phenol in the energy region $0-50 \mathrm{eV}$. Up (cyan) triangles: 1ch-sep; full (black) curve: our best SMCPP channel coupling results for each interval of energy (see text for every energy interval we used the same symbols as in Fig. 10); thin dashed (green): present IAM-SCAR calculation; filled (dark green) squares: experimental results of Ref. 38 for benzene. SMCPP results presented in this figure were obtained in a calculation without use of the Born-closure procedure (see Sec. IX for details).

to properly identify the spurious features from the physical features, we needed a larger number of energy points. At $25 \mathrm{eV}$, we could see that the calculations are quite unstable going from (13ch-sep or 28ch-sep) to (23ch-sep or 33ch-sep). By narrowing the grid of energies, we found a strong and narrow trough around $25 \mathrm{eV}$, due to some spurious closed channel in our CSF space that should in fact be open. Similar features are also seen at 10, 12, and $20 \mathrm{eV}$ impact energies. The latter is due to the threshold of a triplet pseudostate state that opens at $19.75 \mathrm{eV}$ (see Table I). Other narrow spikes and troughs could be found, and in order to properly identify the spurious features from the physical features, we would need a much larger number of points, which would make the calculation very expensive. A similar study (same symbols) is shown in Fig. 11 for our elastic ICS in comparison with the experimental data of Cho et al. ${ }^{38}$ for benzene. Above $3 \mathrm{eV}$, the integral cross sections with and without closure are very close to each other (consistent with phenol's small dipole moment) and only the latter are included for comparison with the experiments for benzene. The agreement here between our theoretical and the experimental elastic ICS is as good as the agreement found for the MTCS.

Figure 12 presents our TCS for electron-phenol scattering from 5 to $50 \mathrm{eV}$ along with the experimental benzene data of Sueoka ${ }^{40}$ and Mozejko et al. ${ }^{41}$ Our SMCPP cross section when summed with the ionization cross section obtained with the BEB model is in very good agreement with present TCS experimental data. The agreement between the SMCPP TCSs obtained with $33 \mathrm{ch}$-sep, full (black) line, and 1ch-sep, full (cyan) line connecting up-triangles, reveals that the latter cannot give good elastic cross sections. As we have discussed previously in the ethylene study, ${ }^{4}$ the present application also shows that as we open more channels, the flux is distributed among them, decreasing substantially the elastic cross sections (clearly noted in our DCSs figures). The 33ch-sep SMCPP

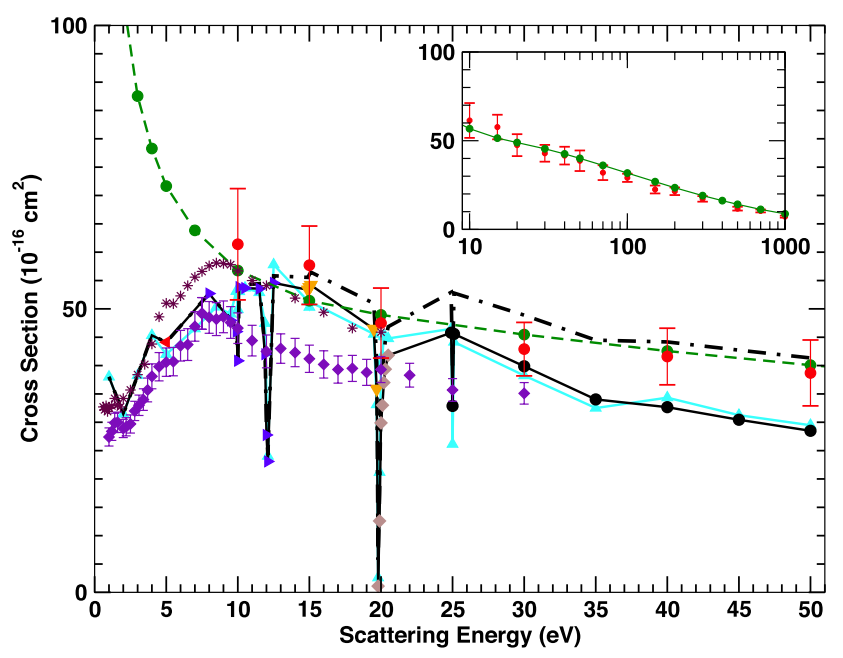

FIG. 12. TCS for phenol in the energy region 0-50 eV. The SMCPP calculations were obtained from Eq. (8). Up (cyan) triangles: 1ch-sep; full (black) line: connecting our best SMCPP channel coupling results for each interval of energy (see text for every energy interval we used the same symbols as in Figs. 10 and 11); thick dotted-dashed line: our best SMCPP TCS plus total ionization cross sections using the BEB approximation; thin dashed line with filled (green) circles: TCS of the IAM-SCAR method; filled (violet) diamonds, (brown) stars, and filled (red) circles: results of the benzene TCS experiments of Ref. 40, of Ref. 41, and present phenol TCS data. The inset shows our theoretical TCS obtained with the IAM-SCAR method and our present experimental phenol TCS data for higher energies. SMCPP results presented in this figure were obtained in a calculation without use of the Born-closure procedure (see Sec. IX for details).

TCS was obtained with the optical theorem, Eq. (8), without closure. If we add the ICSs, channel by channel, as in Eq. (9), and include the BEB result then the resulting TCS is less than $3 \%$ larger than the one obtained by Eq. (8) for energies above $5 \mathrm{eV}$. This is a nice level of self-consistency from the two approaches to derive a SMCPP-based TCS. At very low energies (below $3 \mathrm{eV}$ ), our SMCPP-based TCS does not present a strong increase, shown by the present IAM-SCAR TCSs, which is characteristic of a polar molecule since we have not included the contribution due to the long range dipole potential through the Born closure. The present IAM-SCAR calculated TCS is also in good accord with our measured phenol data, to within the uncertainties on the data, and over the common energy range between 10 and $50 \mathrm{eV}$. Included as an inset of Fig. 12 is a plot showing the higher-energy TCS behavior. As expected, our measured TCS phenol data are in good accord with our IAM-SCAR results at the higher energies.

\section{A LITTLE COMMENT ON THE CHOICE OF $\boldsymbol{\ell}_{\max }$}

Since the issue of convergence of the cross sections in terms of the parameter $\ell_{\max }$ permeates all discussion of the results presented above, we believe that it is appropriate to introduce a complementary comment in order to put the subject on a more general perspective. The Born-closure affects basically the forward scattering. Therefore, for scattering angles above $30^{\circ}-40^{\circ}$, the SMCPP+closure DCSs would be approximately equal to the SMCPP itself (and comparable to experiments). So, Figures 2, 3, and 9 would continue showing convergent results only for $\ell_{\max }=10$ even if we applied the 
Born-closure scheme. We feel confident in applying the Bornclosure strategy when, by incrementing the value of $\ell_{\max }$, only the forward scattering of the SMCPP DCS is affected. For elastic DCSs, this is not the case even for $10 \mathrm{eV}$, as can be seen in Figure 9. In order to be sure that the Born-closure scheme with $\ell_{\max }=4$ could be the right choice, we need an argument that only the higher partial waves of the SMCPP are wrong and therefore deserve to be deleted and replaced by the first Born approximation. Another possibility would be that as we open more and more channels, the lower partial wave contributions would converge and the higher partial wave contribution would decrease. Unfortunately, the whole spectra of partial wave contributions decrease all together with the flux distribution. Therefore, considering the excellent agreement with the experimental data for Benzene in the elastic channel (with multichannel effects and $\ell_{\max }=10$ ), we decided to publish only the DCSs obtained with the converged (backward scattering) SMCPP results, which produces ICS, MTCS, and TCS very little affected by the Born-closure procedure.

\section{CONCLUSIONS}

We have reported experimental and theoretical cross sections for scattering of low-energy electrons by phenol. The SMCPP method was employed to calculate elastic differential, integral, momentum transfer, and total cross sections according to the so-called $N_{\text {open }}$ ch-sep level of approximation. More specifically, up to thirty three electronic states of phenol, obtained within a single excitation configuration-interaction description, were included in the open-channel space. Through the use of this channel coupling scheme, we have performed a detailed analysis of the influence of multichannel coupling effects on the DCS results. As a general trend, we found that the probability flux into the elastic channel decreases as more channels were included in the calculation, leading to a substantial reduction on the magnitude of the corresponding cross sections. This effect becomes even more important for higher impact energies, as expected. The IAM-SCAR method was also used to compute DCSs for energies above $10 \mathrm{eV}$, and the agreement with the SMCPP results was improved as the energy increased. The SMCPP cross sections obtained were in very good agreement with the experimental data measured by Cho et al. for benzene, ${ }^{38}$ both in terms of magnitude and angular distributions.

Integral and momentum transfer cross sections for elastic electron scattering by phenol, obtained with the SMCPP and the IAM-SCAR methods, were compared to the measurements performed for benzene by Cho et al. ${ }^{38}$ The overall good agreement with that experimental data clearly indicates that the inclusion of multichannel coupling effects (from the $3 \mathrm{ch}$ sep up to the 33ch-sep level of approximation) was essential for an adequate description of the scattering process under consideration, especially if compared to the cross sections (DCSs, MTCSs and ICSs) obtained at the 1ch-sep level of approximation, where only the elastic channel was included in the scattering calculation.

The present total cross sections in the energy range from 10 to $50 \mathrm{eV}$ included measurements as well as calculated results obtained by using the IAM-SCAR and the SMCPP (sum of elastic+inelastic+ionization contributions) methods. All these results are in good agreement with each other within the uncertainties associated to the experimental data. In addition, our measured data and IAM-SCAR results at higher energies were also found to be in excellent accord.

\section{ACKNOWLEDGMENTS}

The authors acknowledge support from the Brazilian agency Conselho Nacional de Desenvolvimento Científico e Tecnológico (CNPq). R.F.da.C., M.T.do.N.V., E.M.de.O and M.A.P.L. acknowledge support from Fundação de Amparo à Pesquisa do Estado de São Paulo (FAPESP). The present SMCPP calculations were performed at IFGW-UNICAMP, LCPAD-UFPR, and LFTC-DFis-UFPR. M.H.F.B. acknowledges computational support from Professor Carlos de Carvalho. D.B.J. and M.J.B. acknowledge the Australian Research Council (ARC) for some financial support, in particular D.B.J. thanks the ARC for a Discovery Early Career Researcher Award. M.J.B. also thanks CNPq for his Special Visiting Professor award at the Federal University of Juiz de Fora. P.L.-V. acknowledges support from the Portuguese Foundation for Science and Technology, FCT-MEC though research Grant Nos. PEst-OE/FIS/UI0068/2014 and PTDC/FIS-ATO/1832/2012.

${ }^{1}$ M. Tashiro, J. Chem. Phys. 129, 164308 (2008).

${ }^{2}$ Z. Masín, J. D. Gorfinkiel, D. B. Jones, S. M. Bellm, and M. J. Brunger, J. Chem. Phys. 136, 144310 (2012).

${ }^{3}$ T. N. Rescigno and A. E. Orel, Phys. Rev. A 88, 012703 (2013).

${ }^{4}$ R. F. da Costa, M. H. F. Bettega, M. T. do N. Varella, E. M. de Oliveira, and M. A. P. Lima, Phys. Rev. A 90, 052707 (2014).

${ }^{5}$ R. F. C. Neves, D. B. Jones, M. C. A. Lopes, K. L. Nixon, G. B. da Silva, H. V. Duque, E. M. de Oliveira, R. F. da Costa, M. T. do N. Varella, M. H. F. Bettega, M. A. P. Lima, K. Ratnavelu, G. García, and M. J. Brunger, J. Chem. Phys. 142, 104305 (2015); for technological applications of phenol, see the introduction of this paper and references therein

${ }^{6}$ K. Takatsuka and V. McKoy, Phys. Rev. A 24, 2473 (1981); 30, 1734 (1984).

${ }^{7}$ J. S. dos Santos, R. F. da Costa, and M. T. do N. Varella, J. Chem. Phys. 136, 084307 (2012).

${ }^{8}$ M. H. F. Bettega, L. G. Ferreira, and M. A. P. Lima, Phys. Rev. A 47, 1111 (1993).

${ }^{9}$ R. F. da Costa, F. J. da Paixão, and M. A. P. Lima, J. Phys. B: At., Mol. Opt. Phys. 37, L129 (2004).

${ }^{10}$ M. A. P. Lima, T. L. Gibson, K. Takatsuka, and V. McKoy, Phys. Rev. A 30, 1741 (1984).

${ }^{11}$ E. M. de Oliveira, R. F. da Costa, S. dA. Sanchez, A. P. P. Natalense, M. H. F. Bettega, M. A. P. Lima, and M. T. do N. Varella, Phys. Chem. Chem. Phys. 15, 1682 (2013).

${ }^{12}$ Y.-K. Kim and M. E. Rudd, Phys. Rev. A 50, 3954 (1994).

${ }^{13}$ Y.-K. Kim, W. Hwang, N. M. Weinberger, M. A. Ali, and M. E. Rudd, J. Chem. Phys. 106, 1026 (1997); 106, 9602 (1997); H. Nishimura, W. M. Huo, M. A. Ali, and Y.-K. Kim, ibid. 110, 3811 (1999).

${ }^{14}$ E. M. de Oliveira, S. dA. Sanchez, M. H. F. Bettega, A. P. P. Natalense, M. A. P. Lima, and M. T. do N. Varella, Phys. Rev. A 86, 020701(R) (2012).

${ }^{15}$ D. B. Jones, G. B. da Silva, R. F. C. Neves, H. V. Duque, L. Chiari, E. M. de Oliveira, M. C. A. Lopes, R. F. da Costa, M. T. do N. Varella, M. H. F. Bettega, M. A. P. Lima, and M. J. Brunger, J. Chem. Phys. 141, 074314 (2014).

${ }^{16}$ CRC Handbook of Chemistry and Physics, 79th ed., edited by D. R. Lide (CRC, Boca Raton, 1998).

${ }^{17}$ M. W. Schmidt, K. K. Baldridge, J. A. Boatz, S. T. Elbert, M. S. Gordon, J. H. Jensen, S. Koseki, N. Matsunaga, K. A. Nguyen, S. J. Su, T. L. Windus, M. Dupuis, and J. A. Montgomery, J. Comput. Chem. 14, 1347 (1993).

${ }^{18}$ M. A. P. Lima, L. M. Brescansin, A. J. R. da Silva, C. L. Winstead, and V. McKoy, Phys. Rev. A 41, 327 (1990). 
${ }^{19}$ See http://cccbdb.nist.gov/ for the first ionization potential of phenol.

${ }^{20}$ G. B. da Silva, R. F. C. Neves, L. Chiari, D. B. Jones, E. Ali, D. H. Madison, C. G. Ning, K. L. Nixon, M. C. A. Lopes, and M. J. Brunger, J. Chem. Phys. 141, 124307 (2014).

${ }^{21}$ M. C. Fuss, A. G. Sanz, F. Blanco, J. C. Oller, P. Limão-Vieira, M. J. Brunger, and G. García, Phys. Rev. A 88, 042702 (2013).

${ }^{22}$ A. G. Sanz, M. C. Fuss, F. Blanco, J. D. Gorfinkiel, D. Almeida, F. Ferreira da Silva, P. Limão-Vieira, M. J. Brunger, and G. García, J. Chem. Phys. 139, 184310 (2013).

${ }^{23}$ H. Kato, A. Suga, M. Hoshino, F. Blanco, G. García, P. Limão-Vieira, M. J. Brunger, and H. Tanaka, J. Chem. Phys. 136, 134313 (2012).

${ }^{24}$ H. Kato, K. Anzai, T. Ishihara, M. Hoshino, F. Blanco, G. García, P. LimãoVieira, M. J. Brunger, S. J. Buckman, and H. Tanaka, J. Phys. B: At., Mol. Opt. Phys. 45, 095204 (2012).

${ }^{25}$ M. C. Fuss, A. G. Sanz, F. Blanco, P. Limão-Vieira, M. J. Brunger, and G. García, Eur. Phys. J. D 68, 161 (2014).

${ }^{26}$ M. Hoshino, M. Horie, H. Kato, F. Blanco, G. García, P. Limão-Vieira, J. P. Sullivan, M. J. Brunger, and H. Tanaka, J. Chem. Phys. 138, 214305 (2013).

${ }^{27}$ M. E. Riley and D. G. Truhlar, J. Chem. Phys. 63, 2182 (1975).

${ }^{28}$ X. Z. Zhang, J. F. Sun, and Y. F. Liu, J. Phys. B: At., Mol. Opt. Phys. 25, 1893 (1992).
${ }^{29}$ G. Staszewska, D. W. Schwenke, D. Thirumalai, and D. G. Truhlar, Phys. Rev. A 28, 2740 (1983).

${ }^{30}$ F. Blanco and G. García, Phys. Lett. A 330, 230 (2004).

${ }^{31}$ F. Blanco and G. García, J. Phys. B: At., Mol. Opt. Phys. 42, 145203 (2009).

${ }^{32}$ A. Jain, J. Phys. B: At., Mol. Opt. Phys. 21, 905 (1988).

${ }^{33}$ J. R. Brunton, L. R. Hargreaves, S. J. Buckman, G. García, F. Blanco, O. Zatsarinny, K. Bartschat, and M. J. Brunger, Chem. Phys. Lett. 55, 568-569 (2013).

${ }^{34}$ J. R. Brunton, L. R. Hargreaves, T. M. Maddern, S. J. Buckman, G. García, F. Blanco, O. Zatsarinny, K. Bartschat, D. B. Jones, G. B. da Silva, and M. J. Brunger, J. Phys. B: At., Mol. Opt. Phys. 46, 245203 (2013).

${ }^{35}$ P. Palihawadana, J. P. Sullivan, S. J. Buckman, Z. Mašín, J. D. Gorfinkiel, F. Blanco, G. García, and M. J. Brunger, J. Chem. Phys. 139, 014308 (2013).

${ }^{36}$ J. Setina, Metrologia 36, 623 (1999).

${ }^{37}$ M. J. Brunger and S. J. Buckman, Phys. Rep. 357, 215 (2002).

${ }^{38}$ H. Cho, R. J. Gulley, K. Sunohara, M. Kitajima, L. J. Uhlmann, H. Tanaka, and S. J. Buckman, J. Phys. B: At., Mol. Opt. Phys. 34, 1019 (2001).

${ }^{39}$ G. L. C. de Souza, A. S. dos Santos, R. R. Lucchese, L. E. Machado, L. M. Brescansin, H. V. Manini, I. Iga, and M.-T. Lee, Chem. Phys. 393, 19 (2012).

${ }^{40}$ O. Sueoka, J. Phys. B: At., Mol. Opt. Phys. 21, L631 (1988).

${ }^{41}$ P. Mozejko, G. Kasperski, G. P. Karwasz, C. Szmytkowski, R. S. Brusa, and A. Zecca, Chem. Phys. Lett. 257, 309 (1996). 